\title{
Commensurate Scale Relations in Quantum Chromodynamics 6
}

\author{
Stanley J. Brodsky \\ Stanford Linear Accelerator Center \\ Stanford University, Stanford, California 94309 \\ Hung Jung Lu \\ Department of Physics, University of Maryland \\ College Park, Maryland 20742
}

(September 24, 2018)

\begin{abstract}
We use the BLM method to show that perturbatively-calculable observables in QCD, including the annihilation ratio $R_{e^{+} e^{-}}$, the heavy quark potential, and radiative corrections to structure function sum rules can be related to each other without renormalization scale or scheme ambiguity. The commensurate scale relations connecting the effective charges for observables $A$ and $B$ have the form $\alpha_{A}\left(Q_{A}\right)=\alpha_{B}\left(Q_{B}\right)\left(1+r_{A / B} \frac{\alpha_{B}}{\pi}+\cdots\right)$, where the coefficient $r_{A / B}$ is independent of the number of flavors $f$ contributing to coupling constant renormalization. The ratio of scales $Q_{A} / Q_{B}$ is unique at leading order and guarantees that the observables $A$ and $B$ pass through new quark thresholds at the same physical scale. We also show that the commensurate scales satisfy the renormalization group transitivity rule which ensures that predictions in PQCD are independent of the choice of an intermediate renormalization
\end{abstract}

\footnotetext{
*Work partially supported by the Department of Energy, contract DE-AC03-76SF00515 and contract DE-FG02-93ER-40762.
} 
scheme $C$. In particular, scale-fixed predictions can be made without reference to theoretically-constructed renormalization schemes such as $\overline{\mathrm{MS}}$. QCD can thus be tested in a new and precise way by checking that the observables track both in their relative normalization and in their commensurate scale dependence.

The generalization of the BLM procedure to higher order assigns a different renormalization scale for each order in the perturbative series. The scales are determined by a systematic resummation of running coupling constant effects. The application of this procedure to relate known physical observables in QCD gives surprisingly simple results. In particular, we find that up to light-by-light type corrections, all terms involving $\zeta_{3}, \zeta_{5}$ and $\pi^{2}$ in the relation between the annihilation ratio $R_{e^{+} e^{-}}$and the Bjorken sum rule for polarized electroproduction are automatically absorbed into the renormalization scales. The final series has simple coefficients which are independent of color: $\widehat{\alpha}_{g_{1}}(Q)=\widehat{\alpha}_{R}\left(Q^{*}\right)-\widehat{\alpha}_{R}^{2}\left(Q^{* *}\right)+\widehat{\alpha}_{R}^{3}\left(Q^{* * *}\right)$, where $\widehat{\alpha}=\left(3 C_{F} / 4 \pi\right) \alpha$. The coefficients in the commensurate scale relation can be identified with those obtained in conformally-invariant gauge theory.

11.10.Gh, 11.15.Bt, 12.38.Bx, 13.65.+i

Typeset using REVTEX 


\section{INTRODUCTION}

One of the most serious difficulties preventing precise tests of QCD is the scale ambiguity of its perturbative predictions. Consider a measurable quantity such as $\rho=R_{e^{+} e^{-}}(s)-3 \Sigma e_{q}^{2}$. The PQCD prediction is of the form

$$
\rho=r_{0} \alpha_{s}(\mu)\left[1+r_{1}(\mu) \frac{\alpha_{s}(\mu)}{\pi}+r_{2}(\mu) \frac{\alpha_{s}^{2}(\mu)}{\pi^{2}}+\cdots\right] .
$$

Here $\alpha_{s}(\mu)=g_{s}^{2} / 4 \pi$ is the renormalized coupling defined in a specific renormalization scheme such as $\overline{\mathrm{MS}}$, and $\mu$ is a particular choice of renormalization scale. Since $\rho$ is a physical quantity, its value must be independent of the choice of $\mu$ as well as the choice of renormalization scheme. Nevertheless, since we only have truncated PQCD predictions to a given order $\alpha_{s}^{N}$, the predictions do depend on $\mu$. In the specific case of $R_{e^{+} e^{-}}$, where we have predictions [1:2] through order $\alpha_{s}^{3}$, the sensitivity to $\mu$ has been shown to be less than $10 \%$ over a large range of $\ln \mu$ [2]. However, in the case of the hadronic beauty production cross section $\left(d \sigma / d p_{T}^{2}\right)(\bar{p} p \rightarrow B+X)$, which has been computed to next-to-leading order in $\alpha_{s}$, the prediction [3] for the normalization of the heavy quark $p_{T}$ distribution at hadron colliders

ranges over a factor of 4 if one chooses one "physical value" such as $\mu=\frac{1}{4} \sqrt{m_{B}^{2}+p_{T}^{2}}$ rather than an equally well motivated choice $\mu=\sqrt{m_{B}^{2}+p_{T}^{2}}$.

There is, in fact, no consensus on how to estimate the theoretical error due to the scale ambiguity, what constitutes a reasonable range of physical values, or indeed how to identify what the central value should be. Even worse, if we consider the renormalization scale $\mu$ as totally arbitrary, the next-to-leading coefficient $r_{1}(\mu)$ in the perturbative expansion can take on the value zero or any other value. Thus it is difficult to assess the convergence of the truncated series, and finite-order analyses cannot be meaningfully compared to experiment.

The $\mu$ dependence of the truncated prediction $\rho_{N}$ is often used as a guide to assess the accuracy of the perturbative prediction, since this dependence reflects the presence of the uncalculated terms. However, the scale dependence of $\rho_{N}$ only reflects one aspect of the total series. This point has also been recently emphasized by Maxwell et al. [4]. For example, 
consider the orthopositronium $J^{P C}=1^{--}$decay rate computed in quantum electrodynamics: $\Gamma=\Gamma_{0}[1-10.3(\alpha / \pi)+\cdots]$. The large next-to-leading coefficient, $r_{1}=10.3$ shows that there is important new physics beyond Born approximation. The magnitude of the higher order terms in the decay rate is not related to the renormalization scale since the QED coupling $\alpha$ does not run appreciably at the momentum transfers associated with positronium decay.

Thus we have a difficult dilemma: If we take $\mu$ as an unset parameter in PQCD predictions, then we have no reliable way to assess the accuracy of the truncated series or the parameters extracted from comparison with experiment. If we guess a value for $\mu$ and its range, then we are left with a prediction without an objective guide to its theoretical precision. The problem of the scale ambiguity is compounded in multi-scale problems where several plausible physical scales enter.

In fact three quite distinct methods to set the renormalization scale in PQCD have been proposed in the literature:

1. Fastest Apparent Convergence (FAC) [5]. This method chooses the renormalization scale $\mu$ so that the next-to-leading order coefficient vanishes: $r_{1}(\mu)=0$.

2. The Principle of Minimum Sensitivity (PMS) [6]. In this procedure, one argues that the best scale is the one that minimizes the scale dependence of the truncated prediction $\rho_{N}$, since that is a characteristic property of the entire series. Thus in this method one chooses $\mu$ at the stationary point $d \rho_{N} / d \mu=0$.

3. Brodsky-Lepage-Mackenzie (BLM) [7]. In the BLM scale-fixing method, the scale is chosen such that the coefficients $r_{i}$ are independent of the number of quark flavors $f$ renormalizing the gluon propagators. In leading order, one chooses the scale so that $f$ does not appear in the next-to-leading order coefficient. That is, if $r_{1}(\mu)=r_{10}(\mu)+r_{11}(\mu) f$, where $r_{10}(\mu)$ and $r_{11}(\mu)$ are $f$ independent, then one chooses the scale $\mu$ given by the condition $r_{11}(\mu)=0$. This prescription ensures that, as in quantum electrodynamics, vacuum polarization contributions due to fermion pairs are 
all incorporated into the coupling $\alpha(\mu)$ rather than the coefficients. In the case of non-Abelian theory, the BLM method automatically resums the corresponding gluon as well as quark vacuum polarization contributions since the coupling $\alpha_{s}$ is a function of $\beta_{0} \propto 11-\frac{2}{3} f$.

FIG. 1. The scale $\mu / \sqrt{s}$ according to the BLM (dashed-dotted), PMS (dashed), FAC (full) and $\sqrt{y}$ (dotted) procedures for the three-jet rate in $e^{+} e^{-}$annihilation, as computed by Kramer and Lampe [8]. Notice the strikingly different behavior of the BLM scale from the PMS and FAC scales at low $y$. In particular, the latter two methods predict increasing values of $\mu$ as the jet invariant mass $\mathcal{M}<\sqrt{(y s)}$ decreases.

These scale-setting methods can give strikingly different results in practical applications, For example, Kramer and Lampe have analyzed [8] the application of the FAC, PMS, and BLM methods for the prediction of jet production fractions in $e^{+} e^{-}$annihilation in PQCD. Jets are defined by clustering particles with invariant mass less than $\sqrt{y s}$, where $y$ is the resolution parameter and $\sqrt{s}$ is the total center-of-mass energy. Physically, one expects the renormalization scale $\mu$ to reflect the invariant mass of the jets, that is, $\mu$ should be of order 
$\sqrt{y s}$. For example, in the analogous problem in QED, the maximum virtuality of the photon jet which sets the argument of the running coupling $\alpha(Q)$ cannot be larger than $\sqrt{y s}$. Thus one expects $\mu$ to decrease as the resolution parameter $y \rightarrow 0$. However, the scales chosen by the FAC and PMS methods do not reproduce this behavior (see Fig. 1): The predicted scales $\mu_{P M S}(y)$ and $\mu_{F A C}(y)$ rise without bound at small values for the jet fraction $y$. On the other hand, the BLM scale has the correct physical behavior as $y \rightarrow 0$. Since the argument of the running coupling becomes small using the BLM method, standard QCD perturbation theory in $\alpha_{s}\left[\mu_{B L M}(y)\right]$ will not be convergent in the low $y$ domain [9]. In contrast, the scales chosen by PMS and FAC give no sign that the perturbative results break down in the soft region.

In this paper we shall use the BLM method to show that all perturbatively-calculable observables in QCD, including the annihilation ratio $R_{e^{+} e^{-}}\left(Q^{2}\right)$, the heavy quark potential, and the radiative corrections to the Bjorken sum rule can be related to each other at fixed relative scales. The "commensurate scale relation" for observables $A$ and $B$ in terms of their effective charges has the form

$$
\alpha_{A}\left(Q_{A}\right)=\alpha_{B}\left(Q_{B}\right)\left(1+r_{A / B} \frac{\alpha_{B}}{\pi}+\cdots\right)
$$

The ratio of the scales $\lambda_{A / B}=Q_{A} / Q_{B}$ is chosen so that the coefficient $r_{A / B}$ is independent of the number of flavors $f$ contributing to coupling constant renormalization. This guarantees that the effective charges for the observables $A$ and $B$ pass through new quark thresholds at the same physical scale. We shall show that the value of $\lambda_{A / B}$ is unique at leading order, and that the relative scales satisfy the transitivity rule [10]

$$
\lambda_{A / B}=\lambda_{A / C} \lambda_{C / B}
$$

This is equivalent to the group property defined by Peterman and Stückelberg [11] which ensures that predictions in PQCD are independent of the choice of an intermediate renormalization scheme $C$ 阷. In particular, scale-fixed predictions can be made without reference to theoretically-constructed renormalization schemes such as $\overline{\mathrm{MS}}$; QCD can thus be tested by 
checking that the observables track both in their relative normalization and commensurate scale dependence. 12]

\section{COMMENSURATE SCALE RELATIONS}

It is interesting that the task of setting the renormalization scale has never been considered a problem or ambiguity in perturbative QED. For example, the leading-order parallelhelicity amplitude electron-electron scattering has the form

$$
\mathcal{M}_{e e \rightarrow e e}(++;++)=\frac{8 \pi s}{t} \alpha(t)+\frac{8 \pi s}{u} \alpha(u) .
$$

Here $\alpha(Q)=\alpha\left(Q_{0}\right) /\left(1-\Pi\left[Q^{2}, Q_{0}^{2}, \alpha\left(Q_{0}\right)\right]\right)$ is the QED running coupling which sums all vacuum polarization insertions $\Pi$ into the renormalized photon propagator. The value $\alpha(0)$ is conventionally normalized by Coulomb scattering at $t=-Q^{2}=0$. Notice that both physical scales $t$ and $u$ appear as arguments of the running coupling constant in the various terms contributing to the scattering cross section; if one chooses any other scale for the running coupling, in either the direct or crossed graph amplitude, then one generates a spurious geometric series in $f(\alpha / \pi) \ln \left(-t / \mu^{2}\right)$ or $f(\alpha / \pi) \ln \left(-u / \mu^{2}\right)$, where $f$ represents the number of fermions contributing to the vacuum polarization of the photon propagator.

In general, the "skeleton" expansion of Feynman amplitudes in QED guarantees that all dependence of an observable on the variable $f$ is summed into the running coupling constant; the coefficients in QED perturbation series are thus always $f$-independent once the proper scale in $\alpha$ has been set. Note that the variable $f$ is defined to count only vacuum polarization insertions, not light-by-light loops, since such contributions do not contribute to the coupling constant renormalization in QED.

The use of the running coupling constant $\alpha(Q)$ in QED allows one to sum in closed form all proper and improper vacuum polarization insertions to all orders, thus going well beyond ordinary perturbation theory. For example, consider the perturbative series for the lepton magnetic anomalous moment: 


$$
a_{\ell}=\frac{\alpha\left(Q^{*}\right)}{2 \pi}+r_{2} \frac{\alpha^{2}\left(Q^{* *}\right)}{\pi^{2}}+r_{3} \frac{\alpha^{3}\left(Q^{* * *}\right)}{\pi^{3}}+\cdots
$$

the values $Q^{*}=e^{-5 / 4} m_{\ell}$, etc., can be determined either by the explicit insertion of the running coupling into the integrand of the Feynman amplitude and the mean value theorem, or equivalently, by simply requiring that the coefficients $r_{n}$ be independent of $f$. (Light-by-light scattering contributions are not related to coupling constant renormalization and thus enter explicitly in the order $\alpha^{3}$ coefficient.) Thus the formula for the anomalous moment using the running coupling is form invariant, identical for each lepton $\ell=e, \mu, \tau$, since the dependence on lepton vacuum polarization insertions is implicitly contained in the dependence of the running coupling constant. These examples are illustrations of the general principle that observables such as the anomalous moments can be related to other observables such as the heavy lepton potential $V\left(Q^{2}\right)=-4 \pi \alpha\left(Q^{2}\right) / Q^{2}$, which can be taken as the empirical definition of the on-shell scheme usually used to define $\alpha\left(Q^{2}\right)$.

The same procedure can easily be adapted [7] to non-Abelian theories such as QCD. One of the most useful observables in QCD is the heavy quark potential, since it can be computed in lattice gauge theory from a Wilson loop, and it can be extracted phenomenologically from the heavy quarkonium spectrum. If the interacting quarks have infinite mass, then all radiative correction are associated with the exchange diagrams, rather than the vertex corrections. It is convenient to write the heavy quark potential as $V\left(Q^{2}\right)=-4 \pi C_{F} \alpha_{V}(Q) / Q^{2}$. This defines the "effective charge" $\alpha_{V}\left(Q^{2}\right)$ where by definition the "self-scale" $Q^{2}=-t$ is the momentum transfer squared. The subscript $V$ indicates that the coupling is defined through the potential.

In fact, any perturbatively-calculable physical quantity can be used to define an effective charge [5] by incorporating the entire radiative correction into its definition; for example

$$
R_{e^{+} e^{-}}\left(Q^{2}\right) \equiv R_{e^{+} e^{-}}^{0}\left(Q^{2}\right)\left[1+\frac{\alpha_{R}(Q)}{\pi}\right],
$$

where $R^{0}$ is the Born result and $Q^{2}=s=E_{c m}^{2}$ is the annihilation energy squared. An important result is that all effective charges $\alpha_{A}(Q)$ satisfy the Gell-Mann-Low renormalization group equation with the same $\beta_{0}$ and $\beta_{1}$; different schemes or effective charges only 
differ through the third and higher coefficients of the $\beta$ function. Thus, any effective charge can be used as a reference running coupling constant in QCD to define the renormalization procedure. More generally, each effective charge or renormalization scheme, including $\overline{\mathrm{MS}}$, is a special case of the universal coupling function $\alpha\left(Q, \beta_{n}\right)$ [13. Peterman and Stückelberg have shown [1] that all effective charges are related to each other through a set of evolution equations in the scheme parameters $\beta_{n}$. Physical results relating observables must of course be independent of the choice of any intermediate renormalization scheme.

Let us now consider expanding any observable or effective charge $\alpha_{A}\left(Q_{A}\right)$ in terms of $\alpha_{V}:$

$$
\alpha_{A}\left(Q_{A}\right)=\alpha_{V}(\mu)\left[1+\left(C+D_{V P} f\right) \frac{\alpha_{V}}{\pi}+\cdots\right]
$$

Since $\alpha_{V}$ sums all vacuum polarization contributions by definition, no coefficient in the series expansion in $\alpha_{V}$ can depend on $f$; i.e. all vacuum polarization contributions are already incorporated into the definition of $\alpha_{V}$. Thus we must shift the scale $\mu$ in the argument of $\alpha_{V}$ to the scale [7] $Q_{V}=e^{3 D_{V P}(\mu)} \mu$ :

$$
\alpha_{A}\left(Q_{A}\right)=\alpha_{V}\left(Q_{V}\right)\left[1+r_{1}^{A / V} \frac{\alpha_{V}}{\pi}+\cdots\right]
$$

where $r_{1}^{A / V}=C+(33 / 2) D_{V P}$ is the next-to-leading coefficient in the expansion of the observable $A$ in scheme $V$. Thus the relative scale between the two observables $A$ and $V$, $\lambda_{A / V}=Q_{A} / Q_{V}$, is fixed by the requirement that the coefficients in the expansion in $\alpha_{V}$ scheme are independent of vacuum polarization corrections 佃. Alternatively, one can derive the same result by explicitly integrating the one loop integrals in the calculation of the observable $A$ using $\alpha_{V}\left(\ell^{2}\right)$ in the integrand, where $\ell^{2}$ is the four-momentum transferred squared carried by the gluon. (In practice one only needs to compute the mean-value of $\ell n \ell^{2}=\ln Q_{V}^{2}$ [14.) One can eliminate the $f$ vacuum polarization dependence that appears in the higher order coefficients by allowing a new scale to appear in each order of perturbation theory. In practice, often only the leading order commensurate scale is required in order to test PQCD to good precision. 田 
We can compute other observables $B$ and even effective charges such as $\alpha_{\overline{\mathrm{MS}}}$ as an expansion in $\alpha_{V}$ scheme:

$$
\alpha_{B}\left(Q_{B}\right)=\alpha_{V}\left(Q_{V}\right)\left[1+r_{1}^{B / V} \frac{\alpha_{V}}{\pi}+\cdots\right]
$$

where $Q_{V}=Q_{B} / \lambda_{B / V}$, and again $r_{1}^{B / V}$ must be independent of vacuum polarization contributions. We can now substitute and eliminate $\alpha_{V}\left(Q_{V}\right)$ :

$$
\alpha_{B}\left(Q_{B}\right)=\alpha_{A}\left(Q_{A}\right)\left[1+r_{1}^{B / A} \frac{\alpha_{A}}{\pi}+\cdots\right]
$$

where $Q_{B} / Q_{A}=\lambda_{B / A}=\lambda_{B / V} / \lambda_{A / V}$, and $r_{1}^{B / A}=r_{1}^{B / V}-r_{1}^{A / V}$. Note also the symmetry property $\lambda_{B / A} \lambda_{A / B}=1$. Alternatively, we can compute the commensurate scale $Q_{A}=$ $Q_{B} / \lambda_{B / A}$ directly by requiring $r_{1}^{B / A}$ to be $f$-independent. The result is in agreement with the transitivity rule: the BLM procedure for fixing the commensurate scale ratio between two observables is independent of the intermediate renormalization scheme.

The scale-fixed relation between the heavy quark potential effective charge and $\alpha \overline{\mathrm{MS}}$ is $\alpha_{V}(Q)=\alpha_{\overline{\mathrm{MS}}}\left(e^{-5 / 6} Q\right)\left[1-2\left(\alpha_{\overline{\mathrm{MS}}} / \pi\right)+\cdots\right]$ [7]. (The one-loop calculation of $\alpha_{V}$ in $\overline{\mathrm{MS}}$ scheme is given in Ref. [15].)

The transitivity and symmetry properties of the commensurate scales are the scale transformations of the renormalization "group" as originally defined by Peterman and Stückelberg [11]. The predicted relation between observables must be independent of the order one makes substitutions; i.e. the algebraic path one takes to relate the observables. It is important to note that the PMS method, which fixes the renormalization scale by finding the point of minimal sensitivity to $\mu$, does not satisfy these group properties [10]. The results are chaotic in the sense that the final scale depends on the path of applying the PMS procedure. Furthermore, any method which fixes the scale in QCD must also be applicable to Abelian theories such as QED, since in the limit of small number of colors $N_{C} \rightarrow 0$ the perturbative coefficients in QCD coincide with the perturbative coefficients of an Abelian analog of QCD [\$.

The commensurate scale relations given in Eq. (2.7) provide a new way to test QCD: One can compare two observables by checking that their effective charges agree both in 
normalization and in their scale dependence. The ratio of commensurate scales $\lambda_{A / B}$ is fixed uniquely: it ensures that both observables $A$ and $B$ pass through heavy quark thresholds at precisely the same physical point. Calculations are often performed most advantageously in $\overline{\mathrm{MS}}$ scheme, but all reference to such theoretically-constructed schemes may be eliminated when comparisons are made between observables. This also avoids the problem that one need not expand observables in terms of couplings which have singular or ill-defined functional dependence.

The physical value of the commensurate scale in $\alpha_{V}$ scheme reflects the mean virtuality of the exchanged gluon. However, in other schemes, including $\overline{\mathrm{MS}}$, the argument of the effective charge is displaced from its physical value. The relative scale for a number of observables is indicated in Table I. For example, the physical scale for the branching ratio $\Upsilon \rightarrow \gamma X$ when expanded in terms of $\alpha_{V}$ is $(1 / 2.77) M_{\Upsilon} \sim(1 / 3) M_{\Upsilon}$, which reflects the fact that the final state phase space is divided among three vector systems. (When one expands in $\overline{\mathrm{MS}}$ scheme, the corresponding scale is $0.157 M_{\Upsilon}$.) Similarly, the physical scale appropriate to the hadronic decays of the $\eta_{b}$ is $(1 / 1.67) M_{\eta_{b}} \sim(1 / 2) M_{\eta_{b}}$.

\section{$\underline{\text { Table I }}$}

Leading Order Commensurate Scale Relations

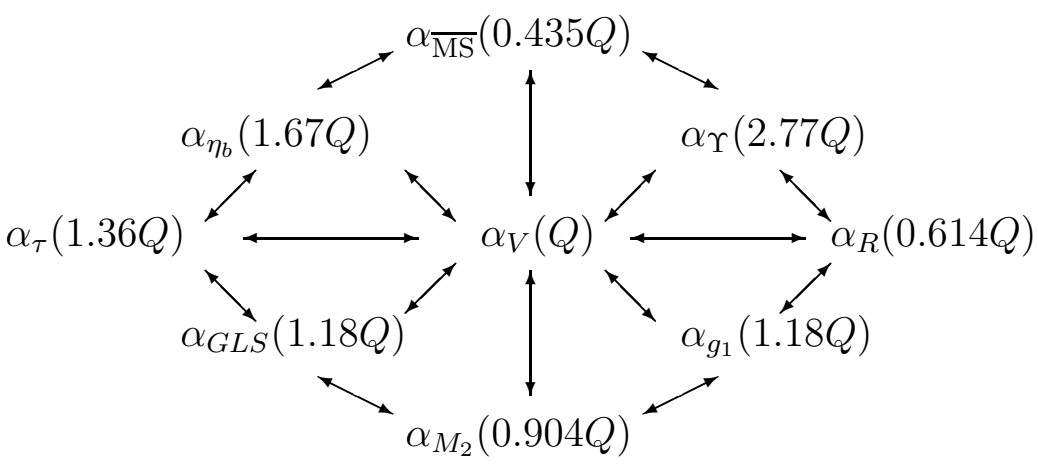

After scale-fixing, the ratio of hadronic to leptonic decay rates for the $\Upsilon$ has the form [7]

$$
\frac{\Gamma(\Upsilon \rightarrow \text { hadrons })}{\Gamma\left(\Upsilon \rightarrow \mu^{+} \mu^{-}\right)}=\frac{10\left(\pi^{2}-9\right)}{81 \pi e_{b}^{2}} \frac{\alpha \frac{3}{\mathrm{MS}}\left(0.157 M_{\Upsilon}\right)}{\alpha_{\mathrm{QED}}^{2}}\left[1-14.0(5) \frac{\alpha_{\overline{\mathrm{MS}}}}{\pi}+\cdots\right]
$$




$$
=\frac{10\left(\pi^{2}-9\right)}{81 \pi e_{b}^{2}} \frac{\alpha_{V}^{3}\left(0.363 M_{\Upsilon}\right)}{\alpha_{\mathrm{QED}}^{2}}\left[1-8.0(5) \frac{\alpha_{V}}{\pi}+\cdots\right] .
$$

Thus, as is the case of positronium decay, the next to leading coefficient is very large, and perturbation theory is not likely to be reliable for this observable. On the other hand, the commensurate scales for the second moment of the non-singlet structure function $M_{2}$ and the effective charges in the Bjorken Sum Rule (and the Gross-Llewellyn-Smith Sum Rule) are not far from the physical value $Q$ when expressed in $\alpha_{V}$ scheme. At large $n$ the commensurate scale for $M_{n}$ is proportional to $1 / \sqrt{n}$, reflecting the fact that the available phase-space for parton emission decreases as $n$ increases. In multiple-scale problems, the commensurate scale can depend on all of the physical invariants. For example, the scale controlling the evolution equation for the non-singlet structure function depends on $x_{B j}$ as well as $Q$ [16]. In the case of inclusive reactions which factorize at leading twist, each structure function, fragmentation function, and subprocess cross section can have its own scale.

The commensurate scale relations between observables can be tested at quite low momentum transfers, even where PQCD relationships would be expected to break down. It is likely that some of the higher twist contributions common to the two observables are also correctly represented by the commensurate scale relations. In contrast, expansions of any observable in $\alpha_{\overline{\mathrm{MS}}}(Q)$ must break down at low momentum transfer since $\alpha_{\overline{\mathrm{MS}}}(Q)$ becomes singular at $Q=\Lambda \overline{\mathrm{MS}}$. (For example, in the 't Hooft scheme where the higher order $\beta_{n}=0$ for $n=2,3, \ldots, \alpha \overline{\mathrm{MS}}(Q)$ has a simple pole at $Q=\Lambda_{\overline{\mathrm{MS}}}$.) The commensurate scale relations allow tests of QCD without explicit reference to schemes such as $\overline{\mathrm{MS}}$. It is thus reasonable to expect that the series expansions are more convergent when one relates finite observables to each other.

\section{NEXT-TO-LEADING-ORDER BLM FORMULAS}

We will now generalize the BLM procedure to relate physical observables beyond leading

order 㫽. As we will see, each order in the expansion will acquire its proper commensurate 
scale. Consider the expansion series of a physical effective charge $\alpha_{1}(Q) / \pi$ in terms of another physical effective charge $\alpha_{2}(Q) / \pi$ 讯

$$
\begin{aligned}
\frac{\alpha_{1}(Q)}{\pi}= & \frac{\alpha_{2}(Q)}{\pi}+\left(A_{12}+B_{12} f\right)\left(\frac{\alpha_{2}(Q)}{\pi}\right)^{2} \\
& +\left(C_{12}+D_{12} f+E_{12} f^{2}\right)\left(\frac{\alpha_{2}(Q)}{\pi}\right)^{3}+\cdots .
\end{aligned}
$$

According to the BLM ansatz, we can reorganize this series and resum higher order terms that are induced by running of the coupling constant effects. We can perform this procedure order by order, and absorb these higher order terms into the renormalization scale of each order. Postponing the justification, we eventually obtain a series of the form

$$
\frac{\alpha_{1}(Q)}{\pi}=\frac{\alpha_{2}\left(Q^{*}\right)}{\pi}+\widetilde{A}_{12}\left(\frac{\alpha_{2}\left(Q^{* *}\right)}{\pi}\right)^{2}+\widetilde{C}_{12}\left(\frac{\alpha_{2}\left(Q^{* * *}\right)}{\pi}\right)^{3}+\cdots
$$

Where the running coupling constant effects have been resummed into the renormalization scales $Q^{*}, Q^{* *}$ and $Q^{* * *}$.

We now analyze the steps involved in this process of resummation. First of all, let us make an important observation: the $B_{12} f$ term and the $E_{12} f^{2}$ term come exclusively from the one-particle-irreducible vacuum polarization graphs with one and two fermion loops. These fermionic contributions belong to the running coupling constant effects, and should be fully absorbed into the renormalization scale $Q^{*}$.

There are also some gluonic contributions in the running coupling constant effects, and consequently some part of $A_{12}, C_{12}$ and $D_{12}$ should also be absorbed into $Q^{*}$. The exact amount is dictated by the behavior of the running coupling constant. The running of $\alpha_{2}(\mu)$ for a general $S U(N)$ group can be characterized by

$$
\begin{aligned}
\frac{\alpha_{2}(\mu)}{\pi}= & \frac{\alpha_{2}\left(\mu_{0}\right)}{\pi}-\frac{1}{4}\left(\frac{11}{3} C_{A}-\frac{4}{3} T f\right) \ln \left(\frac{\mu^{2}}{\mu_{0}^{2}}\right)\left(\frac{\alpha_{2}\left(\mu_{0}\right)}{\pi}\right)^{2} \\
& +\frac{1}{16}\left\{\left(\frac{11}{3} C_{A}-\frac{4}{3} T f\right)^{2} \ln ^{2}\left(\frac{\mu^{2}}{\mu_{0}^{2}}\right)\right. \\
& \left.-\left[\frac{34}{3} C_{A}^{2}-\left(\frac{20}{3} C_{A}+C_{F}\right) T f\right] \ln \left(\frac{\mu^{2}}{\mu_{0}^{2}}\right)\right\}\left(\frac{\alpha_{2}\left(\mu_{0}\right)}{\pi}\right)^{3} \\
& +\cdots
\end{aligned}
$$


In the above formula, $C_{A}=N, C_{F}=\left(N^{2}-1\right) / 2 N$ are the quadratic Casimirs of the adjoint and the fundamental representations. $T$ is the normalization of the trace of generators of the fundamental representation: $\operatorname{Tr}\left(t^{a} t^{b}\right)=T \delta^{a b}$. Conventionally $T$ is chosen to be $1 / 2$.

Let us use $\alpha_{2}(\mu)$ as the strong coupling constant in the calculation of the physical effective charge $\alpha_{1}(Q)$. If we identify the renormalization scale $\mu$ with the momentum transfer of the exchanged gluons in the leading order (LO) Feynman diagrams, and set $\mu_{0}=Q$, the contribution from these diagrams will have the form

$$
\begin{aligned}
{\left[\frac{\alpha_{1}(Q)}{\pi}\right]_{\mathrm{LO}}=} & \frac{\alpha_{2}(Q)}{\pi}-\frac{1}{4}\left(\frac{11}{3} C_{A}-\frac{4}{3} T f\right) L_{1}\left(\frac{\alpha_{2}(Q)}{\pi}\right)^{2} \\
& +\frac{1}{16}\left\{\left(\frac{11}{3} C_{A}-\frac{4}{3} T f\right)^{2} L_{2}\right. \\
& \left.\quad-\left(\frac{34}{3} C_{A}^{2}-\left(\frac{20}{3} C_{A}+C_{F}\right) T f\right) L_{1}\right\}\left(\frac{\alpha_{2}(Q)}{\pi}\right)^{3}+\cdots \\
\equiv & \frac{\alpha_{2}\left(Q^{*}\right)}{\pi} .
\end{aligned}
$$

The quantities $L_{1}$ and $L_{2}$ are two numerical constants, which may be interpreted as the mean-values of the $\ln \left(\mu^{2} / Q^{2}\right)$ and $\ln ^{2}\left(\mu^{2} / Q^{2}\right)$ terms. In general $L_{2} \neq L_{1}^{2}$ due to loop integration or due to the presence of more than one Feynman diagram involving a treelevel gluon propagator. This is the structure of terms that we should absorb in Eq. (3.1). In other words, we can shift the renormalization scale in Eq. (3.1) until we fully absorb those higher order terms with the structure described in Eq. (3.4). To make this step more clear, let us focus on the NLO coefficient of Eq. (3.1), which is given by $A_{12}+B_{12} f$. When we perform a scale shift $Q \rightarrow Q^{*}$, we eliminate the $B_{12} f$ term completely, but at the same time, we also modify the $A_{12}$ term, because the net change to the NLO coefficient is proportional to $\frac{11}{3} C_{A}-\frac{4}{3} T f$, as indicated by the structure of the NLO coefficient in Eq. (3.4). The value of $L_{1}$ is determined by the condition of eliminating the $B_{12} f$ term. Now, proceeding to the NNLO coefficient of Eq. (3.1), which is given by $C_{12}+D_{12} f+E_{12} f^{2}$, we can determined the correct value of $L_{2}$ to eliminate the $E_{12} f^{2}$ term. The modification to the $C_{12}$ and the $D_{12} f$ term is given by the form of the NNLO coefficient in Eq. (3.4). The exact form of the scale shift $Q \rightarrow Q^{*}$ is given later in Eq. (3.15). We should clarify that 
the scale setting happens automatically when we use the running coupling constant inside the Feynman diagram calculation (like the skeleton expansion in QED). The mathematical process here of eliminating the $B_{12} f$ and $E_{12}$ terms allows us to determine the exact amount of the scale shift.

After this first step, our series will look like

$$
\frac{\alpha_{1}(Q)}{\pi}=\frac{\alpha_{2}\left(Q^{*}\right)}{\pi}+\widetilde{A}_{12}\left(\frac{\alpha_{2}\left(Q^{*}\right)}{\pi}\right)^{2}+\left(C_{12}^{\prime}+D_{12}^{\prime} f\right)\left(\frac{\alpha_{2}\left(Q^{*}\right)}{\pi}\right)^{3}+\cdots
$$

The next step to follow is now clear. We should absorb the $D_{12}^{\prime} f$ term into a new renormalization scale $Q^{* *}$ for the NLO term, since the $f$ dependence comes from the vacuum polarization corrections to the NLO term. Naturally part of the $C_{12}^{\prime}$ term should also be absorbed. From the form of the running coupling constant in Eq. (3.3), we know that the absorbed term should be proportional to $\frac{11}{3} C_{A}-\frac{4}{3} T f$. After this procedure we arrive to the form indicated in Eq. (3.2). To the order considered here, we do not have enough information to set the scale $Q^{* * *}$ of the next-to-next-to-leading order (NNLO) coupling constant. A sensible choice is $Q^{* * *}=Q^{* *}$, since this is the renormalization scale after shifting the scales in the second step of the BLM procedure.

In practice, most physical observable in perturbative QCD are computed in the $\overline{\mathrm{MS}}$ scheme, with the running coupling constant fixed at the physical scale of the process. Specifically, if the perturbative series for $\alpha_{1}(Q) / \pi$ and $\alpha_{2}(Q) / \pi$ are

$$
\begin{aligned}
\frac{\alpha_{1}(Q)}{\pi}= & \frac{\alpha_{\overline{\mathrm{MS}}}(Q)}{\pi}+\left(A_{1}+B_{1} f\right)\left(\frac{\alpha_{\overline{\mathrm{MS}}}(Q)}{\pi}\right)^{2} \\
& +\left(C_{1}+D_{1} f+E_{1} f^{2}\right)\left(\frac{\alpha_{\overline{\mathrm{MS}}}(Q)}{\pi}\right)^{3}+\cdots, \\
\frac{\alpha_{2}(Q)}{\pi}= & \frac{\alpha_{\overline{\mathrm{MS}}}(Q)}{\pi}+\left(A_{2}+B_{2} f\right)\left(\frac{\alpha_{\overline{\mathrm{MS}}}(Q)}{\pi}\right)^{2} \\
& +\left(C_{2}+D_{2} f+E_{2} f^{2}\right)\left(\frac{\alpha_{\overline{\mathrm{MS}}}(Q)}{\pi}\right)^{3}+\cdots,
\end{aligned}
$$

then the coefficients $A_{12}, B_{12}, C_{12}, D_{12}$ and $E_{12}$ of Eq. (3.1) are given by 


$$
\begin{aligned}
& A_{12}=A_{1}-A_{2}, \\
& B_{12}=B_{1}-B_{2}, \\
& C_{12}=C_{1}-C_{2}-2\left(A_{1}-A_{2}\right) A_{2}, \\
& D_{12}=D_{1}-D_{2}-2\left(A_{1} B_{2}+A_{2} B_{1}\right)+4 A_{2} B_{2}, \\
& E_{12}=E_{1}-E_{2}-2\left(B_{1}-B_{2}\right) B_{2},
\end{aligned}
$$

and the NLO BLM formulas (for a general $S U(N)$ group) are given by

$$
\begin{aligned}
\widetilde{A}_{12} & =A_{12}+\frac{11}{4} \frac{C_{A}}{T} B_{12}, \\
\widetilde{C}_{12} & =-\frac{3}{16} \frac{C_{A}}{T}\left(7 C_{A}+11 C_{F}\right) B_{12}+C_{12}+\frac{11}{4} \frac{C_{A}}{T} D_{12}+\frac{121}{16} \frac{C_{A}^{2}}{T^{2}} E_{12}, \\
Q^{*} & =Q \exp \left[\frac{3}{2 T} B_{12}+\frac{9}{8 T^{2}}\left(\frac{11}{3} C_{A}-\frac{4}{3} T f\right)\left(B_{12}^{2}-E_{12}\right) \frac{\alpha_{2}(Q)}{\pi}\right], \\
Q^{* *} & =Q \exp \left\{\frac{3}{4 T} \widetilde{A}_{12}^{-1}\left[-\frac{1}{4}\left(5 C_{A}+3 C_{F}\right) B_{12}+D_{12}+\frac{11}{2} \frac{C_{A}}{T} E_{12}\right]\right\} .
\end{aligned}
$$

Notice the presence of $\alpha_{2}(Q) / \pi$ in the expression of $Q^{*}$. In general $Q^{*}$ will itself be a perturbative series in $\alpha_{2}(Q) / \pi$. This fact has first been pointed out in Ref. [7], and also been further explored by Grunberg and Kataev on the extension of the BLM approach [17]. We have exponentiated the perturbative series, since physically the renormalization scale $Q^{*}$ should always be positive. To the order considered here, the scale for the coupling constant in Eq. (3.15) is not well-defined, but can be chosen to be $Q$. This intrinsic uncertainty is similar to the $Q^{* * *}$ scale uncertainty of the NNLO term in Eq. (3.2), and can only be resolved by going to the next-higher order.

For a general gauge field theory, it is interesting to point out that the scale setting procedure described here leads to the correct expansion series coefficients in the "conformal limit" [E耳t. The conformal limit is defined by $\beta_{0}, \beta_{1} \rightarrow 0$, and can be reached, for instance, by adding enough spin-half and scalar quarks. Since all the running coupling effects have been absorbed into the renormalization scales, the scale setting method described here correctly reproduces the expansion coefficients in this limit. It should be pointed out that other scale setting methods in general do not guarantee this feature. 
The application of the scale setting formulas to currently available NNLO QCD quantities gives some very interesting results. The following is a list of some effective charges known to the NNLO.

1) $\alpha_{R}(Q) / \pi$ : the effective charge obtained in total hadronic cross section in $e^{+} e^{-}$annihilation, defined by [1]

$$
R(Q) \equiv 3 \sum_{f} Q_{f}^{2}\left[1+\frac{\alpha_{R}(Q)}{\pi}\right] .
$$

The perturbative series of $\alpha_{R}(Q) / \pi$ is (using $T=1 / 2$ for the trace normalization)

$$
\begin{aligned}
& \frac{\alpha_{R}(Q)}{\pi}=\frac{\alpha_{\overline{\mathrm{MS}}}(Q)}{\pi}+\left(\frac{\alpha_{\overline{\mathrm{MS}}}(Q)}{\pi}\right)^{2}\left[\left(\frac{41}{8}-\frac{11}{3} \zeta_{3}\right) C_{A}-\frac{1}{8} C_{F}+\left(-\frac{11}{12}+\frac{2}{3} \zeta_{3}\right) f\right] \\
&+\left(\frac{\alpha_{\overline{\mathrm{MS}}}(Q)}{\pi}\right)^{3}\left\{\left(\frac{90445}{2592}-\frac{2737}{108} \zeta_{3}-\frac{55}{18} \zeta_{5}-\frac{121}{432} \pi^{2}\right) C_{A}^{2}\right. \\
&+\left(-\frac{127}{48}-\frac{143}{12} \zeta_{3}+\frac{55}{3} \zeta_{5}\right) C_{A} C_{F}-\frac{23}{32} C_{F}^{2} \\
&+ {\left[\left(-\frac{970}{81}+\frac{224}{27} \zeta_{3}+\frac{5}{9} \zeta_{5}+\frac{11}{108} \pi^{2}\right) C_{A}\right.} \\
&\left.+\left(-\frac{29}{96}+\frac{19}{6} \zeta_{3}-\frac{10}{3} \zeta_{5}\right) C_{F}\right] f \\
&+\left(\frac{151}{162}-\frac{19}{27} \zeta_{3}-\frac{1}{108} \pi^{2}\right) f^{2} \\
&+\left.\left(\frac{11}{144}-\frac{1}{6} \zeta_{3}\right) \frac{d^{a b c} d^{a b c}}{C_{F} d(R)} \frac{\left(\sum_{f} Q_{f}\right)^{2}}{\sum_{f} Q_{f}^{2}}\right\} .
\end{aligned}
$$

The term containing $\left(\sum_{f} Q_{f}\right)^{2} / \sum_{f} Q_{f}^{2}$ arises from light-by-light diagrams. The dimension of the quark representation is $d(R)$, which usually is $N$ for $S U(N)$. For QCD we have $d^{a b c} d^{a b c}=40 / 3$. It is interesting to point out that the vector part of the $Z$ hadronic decay width 18 shares the same effective charge as $R(Q)$.

2) $\alpha_{\tau}\left(M_{\tau}\right) / \pi$ : the effective charge obtained from the perturbative hadronic decay rate of the $\tau$ lepton, defined by [1]

$$
R_{\tau} \equiv 3\left[1+\frac{\alpha_{\tau}\left(M_{\tau}\right)}{\pi}\right]
$$

The perturbative series of $\alpha_{\tau}\left(M_{\tau}\right) / \pi$ is

$$
\frac{\alpha_{\tau}(Q)}{\pi}=\frac{\alpha_{\overline{\mathrm{MS}}}(Q)}{\pi}+\left(\frac{\alpha_{\overline{\mathrm{MS}}}(Q)}{\pi}\right)^{2}\left[\left(\frac{947}{144}-\frac{11}{3} \zeta_{3}\right) C_{A}-\frac{1}{8} C_{F}+\left(-\frac{85}{72}+\frac{2}{3} \zeta_{3}\right) f\right]
$$




$$
\begin{aligned}
+\left(\frac{\alpha_{\overline{\mathrm{MS}}}(Q)}{\pi}\right)^{3} & \left\{\left(\frac{559715}{10368}-\frac{2591}{72} \zeta_{3}-\frac{55}{18} \zeta_{5}-\frac{121}{432} \pi^{2}\right) C_{A}^{2}\right. \\
+ & \left(-\frac{1733}{576}-\frac{143}{12} \zeta_{3}+\frac{55}{3} \zeta_{5}\right) C_{A} C_{F}-\frac{23}{32} C_{F}^{2} \\
+ & {\left[\left(-\frac{24359}{1296}+\frac{73}{6} \zeta_{3}+\frac{5}{9} \zeta_{5}+\frac{11}{108} \pi^{2}\right) C_{A}\right.} \\
& \left.+\left(-\frac{125}{288}+\frac{19}{6} \zeta_{3}-\frac{10}{3} \zeta_{5}\right) C_{F}\right] f \\
+ & \left.\left(\frac{3935}{2592}-\frac{19}{18} \zeta_{3}-\frac{1}{108} \pi^{2}\right) f^{2}\right\}
\end{aligned}
$$

The appropriate number of flavors to be used here is $f=3$. There is no light-by-light contributions in $\alpha_{\tau} / \pi$.

3) $\underline{\alpha_{g_{1}}(Q) / \pi}$ : the effective charge obtained from the Bjorken sum rule for polarized electroproduction, defined by [19]

$$
\int_{0}^{1} d x\left[g_{1}^{e p}\left(x, Q^{2}\right)-g_{1}^{e n}\left(x, Q^{2}\right)\right] \equiv \frac{1}{3}\left|\frac{g_{A}}{g_{V}}\right|\left[1-\frac{\alpha_{g_{1}}(Q)}{\pi}\right] .
$$

Notice that there are different normalization conventions in the literature for the polarized structure functions. Here we follow the normalization given in Ref. [19]. For a recent review on this sum rule, see Ref. [20]. The perturbative series of $\alpha_{g_{1}}(Q) / \pi$ is

$$
\begin{aligned}
\frac{\alpha_{g_{1}}(Q)}{\pi}=\frac{\alpha_{\overline{\mathrm{MS}}}(Q)}{\pi}+\left(\frac{\alpha_{\overline{\mathrm{MS}}}(Q)}{\pi}\right)^{2}\left[\frac{23}{12} C_{A}-\frac{7}{8} C_{F}-\frac{1}{3} f\right] \\
+\left(\frac{\alpha_{\overline{\mathrm{MS}}}(Q)}{\pi}\right)^{3}\left\{\left(\frac{5437}{648}-\frac{55}{18} \zeta_{5}\right) C_{A}^{2}+\left(-\frac{1241}{432}+\frac{11}{9} \zeta_{3}\right) C_{A} C_{F}+\frac{1}{32} C_{F}^{2}\right. \\
+\left[\left(-\frac{3535}{1296}-\frac{1}{2} \zeta_{3}+\frac{5}{9} \zeta_{5}\right) C_{A}+\left(\frac{133}{864}+\frac{5}{18} \zeta_{3}\right) C_{F}\right] f \\
\left.+\frac{115}{648} f^{2}\right\} .
\end{aligned}
$$

For $\alpha_{g_{1}} / \pi$ we do not have light-by-light contributions, either.

4) $\underline{\alpha}_{F_{3}}(Q) / \pi$ : the effective charge obtained from the Gross-Llewellyn Smith sum rule, defined by 19

$$
\int_{0}^{1} d x\left[F_{3}^{\bar{\nu} p}\left(x, Q^{2}\right)+F_{3}^{\nu p}\left(x, Q^{2}\right)\right] \equiv 6\left[1-\frac{\alpha_{F_{3}}(Q)}{\pi}\right]
$$


As pointed out in Ref. [19], the effective charge $\alpha_{F_{3}} / \pi$ differ from $\alpha_{g_{1}} / \pi$ only by the lightby-light contributions. The perturbative series of $\alpha_{F_{3}} / \pi$ is given by the perturbative series of $\alpha_{g_{1}} / \pi$ plus the additional term in the following formula.

$$
\frac{\alpha_{F_{3}}(Q)}{\pi}=\frac{\alpha_{g_{1}}(Q)}{\pi}-\left(\frac{\alpha_{\overline{\mathrm{MS}}}(Q)}{\pi}\right)^{3} \frac{d^{a b c} d^{a b c}}{C_{F} N_{C}}\left(-\frac{11}{144}+\frac{1}{6} \zeta_{3}\right) f .
$$

For QCD we have $C_{F}=4 / 3$, the number of colors is $N_{C}=3$, and $d^{a b c} d^{a b c}=40 / 3$. Since the radiative corrections to the Bjorken sum rule are identical to those of the Gross-LlewellynSmith sum rule - up to small corrections of order $\alpha_{s}^{3}\left(Q^{2}\right)$, a basic test of QCD can be made by considering the ratio of the Gross-Llewellyn-Smith and Bjorken sum rules:

$$
R_{G L L S / B j}\left(Q^{2}, \epsilon\right)=\frac{\frac{1}{6} \int_{\epsilon}^{1} d x\left[F_{3}^{\nu p}\left(x, Q^{2}\right)+F_{3}^{\bar{\nu} p}\left(x, Q^{2}\right)\right]}{3\left|\frac{g_{V}}{g_{A}}\right| \int_{\epsilon}^{1} d x\left[g_{1}^{p}\left(x, Q^{2}\right)-g_{1}^{n}\left(x, Q^{2}\right)\right]} .
$$

Since the Regge behavior of the two sum rules is similar, the empirical extrapolation to $\epsilon \rightarrow 0$ should be relatively free of systematic error. Moreover, PQCD predicts

$$
R_{G L L S / B j}\left(Q^{2}, \epsilon \rightarrow 0\right)=1+\mathcal{O}\left(\alpha_{s}^{3}(Q)\right)+\mathcal{O}\left(\frac{\Lambda_{Q C D}^{2}}{Q^{2}}\right),
$$

i.e., hard relativistic corrections to the ratio of the sum rules only enter at three loops. Thus measurements of the ratio of the sum rules could provide a remarkably complication-free test of QCD - any significant deviation from $R_{G L L S / B j}\left(Q^{2}, \epsilon \rightarrow 0\right)=1$ must be due to higher twist effects which should vanish rapidly with increasing $Q^{2}$.

5) $\alpha_{F_{1}}(Q) / \pi$ : the effective charge obtained from the Bjorken sum rule for deep-inelastic neutrino-nucleon scattering, defined by [21]

$$
\int_{0}^{1} d x\left[F_{1}^{\bar{\nu} p}\left(x, Q^{2}\right)-F_{1}^{\nu p}\left(x, Q^{2}\right)\right] \equiv 1-\frac{1}{2} C_{F}\left(\frac{\alpha_{F_{1}}(Q)}{\pi}\right) .
$$

The perturbative series of $\alpha_{F_{1}}(Q) / \pi$ is

$$
\begin{aligned}
\frac{\alpha_{F_{1}}(Q)}{\pi}= & \frac{\alpha_{\overline{\mathrm{MS}}}(Q)}{\pi}+\left(\frac{\alpha_{\overline{\mathrm{MS}}}(Q)}{\pi}\right)^{2}\left[\frac{91}{36} C_{A}-\frac{11}{8} C_{F}-\frac{4}{9} f\right] \\
+\left(\frac{\alpha_{\overline{\mathrm{MS}}}(Q)}{\pi}\right)^{3} & \left\{\left(\frac{8285}{648}+5 \zeta_{3}-10 \zeta_{5}\right) C_{A}^{2}+\left(-\frac{2731}{144}-\frac{91}{3} \zeta_{3}+\frac{95}{2} \zeta_{5}\right) C_{A} C_{F}\right. \\
+ & \left(\frac{313}{32}+\frac{47}{2} \zeta_{3}-35 \zeta_{5}\right) C_{F}^{2}
\end{aligned}
$$




$$
\begin{aligned}
& +\left[\left(-\frac{4235}{1296}+\frac{7}{6} \zeta_{3}-\frac{5}{3} \zeta_{5}\right) C_{A}+\left(\frac{335}{288}-\frac{1}{6} \zeta_{3}\right) C_{F}\right] f \\
& \left.+\frac{155}{648} f^{2}\right\} .
\end{aligned}
$$

For $\alpha_{F_{1}} / \pi$ we do not have light-by-light contributions, either.

As the first example of a beyond leading order commensurate scale relation, we shall express $\alpha_{\tau}\left(M_{\tau}\right) / \pi$ in terms of $\alpha_{R}(Q) / \pi$. The appropriate number of flavors in this case is $f=3$, because $\tau$ decay occurs below the charm threshold. [Incidentally, the light-by-light contribution in $\alpha_{R}(Q) / \pi$ vanishes for the three flavor case.] The application of the NLO BLM formulas leads to the following commensurate scale relation

$$
\begin{aligned}
\frac{\alpha_{\tau}\left(M_{\tau}\right)}{\pi} & =\frac{\alpha_{R}\left(Q^{*}\right)}{\pi} \\
Q^{*} & =M_{\tau} \exp \left[-\frac{19}{24}-\frac{169}{128} \frac{\alpha_{R}\left(M_{\tau}\right)}{\pi}\right] .
\end{aligned}
$$

Notice that all the $\zeta_{3}, \zeta_{5}$ and $\pi^{2}$ terms present in the perturbative series of $\alpha_{R}(Q) / \pi$ and $\alpha_{\tau}\left(M_{\tau}\right) / \pi$ have disappeared when we related these two physical observables directly. Notice also the vanishing NLO and NNLO coefficient in Eq. (3.29). That is, up to the NNLO, the two effective charge are simply related by a BLM scale shift.

As the next example, let us express $\alpha_{g_{1}}(Q) / \pi$ in terms of $\alpha_{R}(Q) / \pi$. We will leave the $f$ dependence explicit. The application of the NLO BLM formulas leads to

$$
\begin{aligned}
\frac{\alpha_{g_{1}}(Q)}{\pi}= & \frac{\alpha_{R}\left(Q^{*}\right)}{\pi}-\frac{3}{4} C_{F}\left(\frac{\alpha_{R}\left(Q^{* *}\right)}{\pi}\right)^{2} \\
& +\left[\frac{9}{16} C_{F}^{2}-\left(\frac{11}{144}-\frac{1}{6} \zeta_{3}\right) \frac{d^{a b c} d^{a b c}}{C_{F} N} \frac{\left(\sum_{f} Q_{f}\right)^{2}}{\sum_{f} Q_{f}^{2}}\right]\left(\frac{\alpha_{R}\left(Q^{* * *}\right)}{\pi}\right)^{3}, \\
Q^{*}= & Q \exp \left[\frac{7}{4}-2 \zeta_{3}+\left(\frac{11}{96}+\frac{7}{3} \zeta_{3}-2 \zeta_{3}^{2}-\frac{\pi^{2}}{24}\right)\left(\frac{11}{3} C_{A}-\frac{2}{3} f\right) \frac{\alpha_{R}(Q)}{\pi}\right], \\
Q^{* *}= & Q \exp \left[\frac{523}{216}+\frac{28}{9} \zeta_{3}-\frac{20}{3} \zeta_{5}+\left(-\frac{13}{54}+\frac{2}{9} \zeta_{3}\right) \frac{C_{A}}{C_{F}}\right] .
\end{aligned}
$$

As explained previously, the scale $Q^{* * *}$ in the above expression can be chosen to be $Q^{* *}$. Notice that aside from the light-by-light contributions, all the $\zeta_{3}, \zeta_{5}$ and $\pi^{2}$ dependencies have been absorbed into the renormalization scales $Q^{*}$ and $Q^{* *}$. Understandably, the $\pi^{2}$ term 
should be absorbed into renormalization scale since it comes from the analytical continuation of QCD coupling constant from the spacelike region to the time-like region. However, at present we do not have an full understanding for the disappearance of the $\zeta_{3}$ and $\zeta_{5}$ terms.

For the three flavor case, or neglecting the light-by-light contribution, the series simplifies to

$$
\widehat{\alpha}_{g_{1}}(Q)=\widehat{\alpha}_{R}\left(Q^{*}\right)-\widehat{\alpha}_{R}^{2}\left(Q^{* *}\right)+\widehat{\alpha}_{R}^{3}\left(Q^{* * *}\right)
$$

where

$$
\begin{aligned}
& \widehat{\alpha}_{g_{1}}(Q)=\frac{3 C_{F}}{4 \pi} \alpha_{g_{1}}(Q), \\
& \widehat{\alpha}_{R}(Q)=\frac{3 C_{F}}{4 \pi} \alpha_{R}(Q) .
\end{aligned}
$$

Observe the asthonishing simplicity of the expansion series in Eq. (3.34). These last formulas suggest that for the general $S U(N)$ group the natural expansion parameter is $\widehat{\alpha}$. The use of $\widehat{\alpha}$ also makes explicit that the same formula is valid for QCD and QED. That is, in the limit $N_{C} \rightarrow 0$ the perturbative coefficients in QCD coincide with the perturbative coefficients of an Abelian analog of QCD «\$].

Broadhurst and Kataev have recently observed a number of interesting relations between $\alpha_{R}(Q)$ and $\alpha_{g_{1}}(Q)$ (the "Seven Wonders") [22]. Our simple result here reinforces the idea of a "secret symmetry" between $\alpha_{R}(Q)$ and $\alpha_{g_{1}}(Q)$. We see that this hidden simplicity is only revealed after the application of the NLO BLM scale setting procedure.

The application of the NLO BLM formulas to related the other effective charges presented here give results with similar simplicity (see Appendix), except for those cases involving $\alpha_{F_{1}}$. For instance, the obtained relation for $\alpha_{\tau}\left(M_{\tau}\right)$ and $\alpha_{F_{1}}$ is

$$
\begin{aligned}
\frac{\alpha_{\tau}\left(M_{\tau}\right)}{\pi}= & \frac{\alpha_{F_{1}}\left(Q^{*}\right)}{\pi}+\frac{5}{4} C_{F}\left(\frac{\alpha_{F_{1}}\left(Q^{* *}\right)}{\pi}\right)^{2} \\
& +\left\{\left(-\frac{43}{12}-\frac{85}{6} \zeta_{3}+\frac{115}{6} \zeta_{5}\right) C_{A}^{2}+\left(10+34 \zeta_{3}-\frac{95}{2} \zeta_{5}\right) C_{A} C_{F}\right. \\
& \left.+\left(-\frac{113}{16}-\frac{47}{2} \zeta_{3}+35 \zeta_{5}\right) C_{F}^{2}\right\}\left(\frac{\alpha_{F_{1}}\left(Q^{* * *}\right)}{\pi}\right)^{3}
\end{aligned}
$$




$$
\begin{aligned}
Q^{*} & =M_{\tau} \exp \left[-\frac{53}{24}+2 \zeta_{3}+\left(-\frac{143}{384}-\frac{7}{3} \zeta_{3}+2 \zeta_{3}^{2}+\frac{\pi^{2}}{24}\right)\left(\frac{11}{3} C_{A}-\frac{2}{3} f\right) \frac{\alpha_{F_{1}}\left(M_{\tau}\right)}{\pi}\right], \\
Q^{* *} & =M_{\tau} \exp \left[-\frac{47}{20}+\frac{28}{5} \zeta_{3}-4 \zeta_{5}+\left(-\frac{1}{2}-\frac{28}{15} \zeta_{3}+\frac{8}{3} \zeta_{5}\right) \frac{C_{A}}{C_{F}}\right] .
\end{aligned}
$$

We see that the $\pi^{2}$ terms is absorbed into the renormalization scale $Q^{*}$, but the resulting coefficients in the expansion series are not as simple as in the previous examples. In particular, we observe that the $\zeta_{3}$ and $\zeta_{5}$ terms persist in the NNLO coefficient, which also has a $C_{A}$ dependence.

When the scale setting formulas are applied to relate physical effective charges to the somewhat artificial $\overline{\mathrm{MS}}$ scheme coupling constant, no comparable simplicity is observed. For instance, the application of the formulas to relate $\alpha_{R}(Q)$ to $\alpha_{\overline{\mathrm{MS}}}(Q)$ leads to

$$
\begin{array}{rl}
\frac{\alpha_{R}(Q)}{\pi}= & \frac{\alpha_{\overline{\mathrm{MS}}}\left(Q^{*}\right)}{\pi}+\tilde{A}_{12} \frac{\alpha_{\overline{\mathrm{MS}}}^{2}\left(Q^{* *}\right)}{\pi}+\tilde{C}_{12} \frac{\alpha \frac{3}{\mathrm{MS}}\left(Q^{* * *}\right)}{\pi} \\
\tilde{A}_{12}= & \frac{C_{A}}{12}-\frac{C_{F}}{8} \\
\tilde{C}_{12}= & \left(-\frac{53}{144}-\frac{11}{4} \zeta_{3}\right) C_{A}^{2}+\left(-\frac{101}{192}+\frac{11}{4} \zeta_{3}\right) C_{A} C_{F} \\
& -\frac{23}{32} C_{F}^{2}+\frac{d^{a b c} d^{a b c}}{3 C_{F} d(R)}\left(\frac{11}{48}-\frac{1}{2} \zeta_{3}\right) \frac{\left(\sum_{f} Q_{f}\right)^{2}}{\sum_{f} Q_{f}^{2}} \\
& \left.+\left(-\frac{119}{288}-\frac{7}{3} \zeta_{3}+2 \zeta_{3}^{2}+\frac{\pi^{2}}{24}\right)\left(\frac{11}{3} C_{A}-\frac{2}{3} f\right) \frac{\alpha_{\overline{\mathrm{MS}}}(Q)}{\pi}\right] \\
Q^{*} & Q \exp \left[-\frac{11}{4}+2 \zeta_{3}\right. \\
Q^{* *}= & Q \exp \left[\frac{\left(-166-80 \zeta_{3}+160 \zeta_{5}\right) C_{A}+\left(111+768 \zeta_{3}-960 \zeta_{5}\right) C_{F}}{16 C_{A}-24 C_{F}}\right] .
\end{array}
$$

These last expressions clearly do not exhibit the simplicity of the cases shown in Eq. (3.29) or Eq. (3.34).

\section{CONCLUSION}

The problem of the scale ambiguity of PQCD predictions has plagued attempts to make reliable and precise tests of the theory. In this paper we have shown how this problem can be avoided by focussing on relations between experimentally-measurable observables. 
The conventional $\overline{\mathrm{MS}}$ renormalization scheme serves simply as an intermediary between observables. For example, consider the entire radiative corrections to the annihilation cross section expressed as the effective charge $\alpha_{R}(Q)$ where $Q=\sqrt{s}$ :

$$
R(Q) \equiv 3 \sum_{f} Q_{f}^{2}\left[1+\frac{\alpha_{R}(Q)}{\pi}\right]
$$

Similarly, we can define the entire radiative correction to the Bjorken sum rule as the effective charge $\alpha_{g_{1}}(Q)$ where $Q$ is the lepton momentum transfer:

$$
\int_{0}^{1} d x\left[g_{1}^{e p}\left(x, Q^{2}\right)-g_{1}^{e n}\left(x, Q^{2}\right)\right] \equiv \frac{1}{3}\left|\frac{g_{A}}{g_{V}}\right|\left[1-\frac{\alpha_{g_{1}}(Q)}{\pi}\right] .
$$

We now use the known expressions to three loops in $\overline{\mathrm{MS}}$ scheme and choose the scales $Q^{*}$ and $Q^{* *}$ to re-sum all quark and gluon vacuum polarization corrections into the running couplings. The value of these scales are the physical values which insure that each observable passes through the heavy quark thresholds at their respective commensurate physical scales. The final result is remarkably simple:

$$
\frac{\alpha_{g_{1}}(Q)}{\pi}=\frac{\alpha_{R}\left(Q^{*}\right)}{\pi}-\left(\frac{\alpha_{R}\left(Q^{* *}\right)}{\pi}\right)^{2}+\left(\frac{\alpha_{R}\left(Q^{* * *}\right)}{\pi}\right)^{3}+\ldots
$$

A fundamental test of QCD is to verify empirically that the observables track in both normalization and shape as given by these relations. The coefficients in the series (aside for a factor of $C_{F}$ which can be absorbed in the definition of $\alpha_{s}$ ) are actually independent of color and are the same in Abelian, non-Abelian, and conformal gauge theory. The nonAbelian structure of the theory is reflected in the scales $Q^{*}$ and $Q^{* *}$. The commensurate scale relations thus provide fundamental tests of QCD which can be made increasingly precise and independent of any scheme or other theoretical convention.

We have also presented in this paper a number of other commensurate scale relations using the extension of the BLM method to the next-to-leading order. We have shown that in each case the application of the NLO BLM formulas to relate known physical observables in QCD leads to results with surprising elegance and simplicity.

In principle, commensurate scale relations allow tests of perturbative QCD with higher and higher precision as the perturbative expansion grows. They also provide a new way 
to specify QCD phenomenology. Because they relate observables, the commensurate scale relations are convention-independent; i.e., independent of the normalization conventions used to define the color $S U(N)$ matrices, etc. Since the ambiguities due to scale and scheme choice have been eliminated, one can ask fundamental questions concerning the nature of the QCD perturbative expansions, e.g., whether the series is convergent or asymptotic due to renormalons, etc. [23]. The precision of the leading twist series will also allow sensitive tests for higher twist contributions to physical observables.

We emphasize that any consistent renormalization scheme, $\overline{\mathrm{MS}}$, MS, MOM, etc., with any arbitrary choice of renormalization scale $\mu$, can be used in the intermediate stages of analysis. The final result, the commensurate scale relation between observables, is guaranteed to be independent of the choice of intermediate renormalization scheme since the BLM procedure satisfies the generalized renormalization group properties of Peterman and Stuckelberg. An important computational advantage is that one only needs to compute the $f$-dependence of the higher order terms in order to specify the lower order scales in the commensurate scale relations. In many cases, the series coefficients in the commensurate scale relations can be determined from the corresponding Abelian theory; i.e. $N_{C} \rightarrow 0$.

The BLM method and the commensurate scale relations presented here can be applied to the whole range of QCD and standard model processes, making the tests of theory much more sensitive. The method should also improve precision tests of electroweak, supersymmetry and other non-Abelian theories. One of the most interesting and important areas of application of commensurate scale relations will be to the hadronic corrections to exclusive and inclusive weak decays of heavy quark systems, since the scale ambiguity in the QCD radiative corrections is at present often the largest component in the theoretical error entering electroweak phenomenology.

The commensurate scale relations for some of the observables discussed in this paper $\left(\alpha_{R}, \alpha_{\tau}, \alpha_{g_{1}}\right.$ and $\left.\alpha_{F_{3}}\right)$ are universal in the sense that the coefficients of $\widehat{\alpha}_{s}$ are independent of color; in fact, they are the same as those for Abelian gauge theory. Thus much information on the structure of the non-Abelian commensurate scale relations can be obtained from much 
simpler Abelian analogs. In fact, in the examples we have discussed here, the non-Abelian nature of gauge theory is reflected in the $\beta$-function coefficients and the choice of secondorder scale $Q^{* *}$. The lack of convergence of the non-Abelian theory such as renormalon behavior could show up as a progressive decrease of the higher order commensurate scales. The coefficients in these commensurate scale relations are simply \pm 1 and 0 and suggest that the underlying relation between observables without light-by-light contributions is possibly a geometric series. Note that the relative correction due to the three loop corrections is $\pm\left(\alpha_{s} / \pi\right)^{2}$ or less than $1 \%$ for $\alpha_{s}<0.3$.

A natural procedure for developing a precision QCD phenomenology is to choose one effective charge as the canonical definition of the QCD coupling, and then predict all other observables in terms of this canonical measure. Ideally, the heavy quark effective charge $\alpha_{V}\left(Q^{2}\right)$ could serve this central role since it can be determined from both the quarkonium spectrum and from lattice gauge theory. However, in order for this effective charge to be useful in practice, it will be necessary to compute the relation of the heavy quark potential to other schemes through three loops. At present, the most precisely theoretically and empirically known effective coupling is $\alpha_{R}\left(Q^{2}\right)$, as determined from the annihilation cross section; thus it is natural to use it as the standard definition.

Alternatively, one can follow historical convention and continue to use the $\overline{\mathrm{MS}}$ scheme as an intermediary between observables. For definiteness, we can define the $\overline{\mathrm{MS}}$ scheme as having all $\beta_{n}=0$ beyond $n=1$. The commensurate scale relations such as Eq. (3.15), (3.16) then unambiguously specify all of the scales $Q^{*}, Q^{* *}$, etc. required to relate $\alpha \overline{\mathrm{MS}}$ to the observables. The intrinsic QCD scale will then be unambiguously encoded as $\Lambda_{\overline{\mathrm{MS}}}$. However, there is am intrinsic disadvantage in using $\alpha \overline{\mathrm{MS}}(Q)$ as an expansion parameter: the function $\alpha_{\overline{\mathrm{MS}}}(Q)$ has a simple pole at $Q=\Lambda_{\overline{\mathrm{MS}}}$, whereas observables are by definition finite.

The BLM scale has also recently been used by Lepage and Mackenzie [14 and their co-workers to improve lattice perturbation theory. By using the BLM method one can eliminate $\alpha_{\text {Lattice }}$ in favor of $\alpha_{V}$ thus avoiding an expansion with artificially large coefficients. The lattice determination, together with the empirical constraints from the heavy quarko- 
nium spectra, promises to provide a well-determined effective charge $\alpha_{V}(Q)$ which could be adopted as the QCD standard coupling.

After one fixes the renormalization scale $\mu$ to the BLM value, it is still useful to compute the logarithmic derivative of the truncated perturbative prediction $d \ell n \rho_{N} / d \ell n \mu$ at the BLM-determined scale. If this derivative is large, or equivalently, if the BLM and PMS scales strongly differ, then one knows that the truncated perturbative expansion cannot be numerically reliable, since the entire series is independent of $\mu$. Note that this is a necessary condition for a reliable series, not a sufficient one, as evidenced by the large coefficients in the positronium and quarkonium decay widths which appear when the scales are set correctly. In the case of the two and three jet decay fractions in $e^{+} e^{-}$annihilation, the BLM and PMS scales strongly differ at low values of the jet discriminant $y$. Thus, by using this criterion, we establish that the perturbation theory must fail in the small $y$ regime, requiring careful resummation of the $\alpha_{s} l n y$ series. (A more detailed discussion of the sensitivity of the jet fractions to scale choice and jet clustering schemes is given in Ref. [9].)

However, if we restrict the analysis to jets with invariant mass $\mathcal{M}<\sqrt{y s}$, with $0.14>$ $y>0.05$, then we have an ideal situation, since both the PMS and FAC scales nearly coincide with the BLM scale when one computes jet ratios in the $\overline{\mathrm{MS}}$ scheme (See Fig. 1.) i.e., the renormalization scale dependence in this case is minimal at the BLM scale, and the computed NLO (next-to-leading order) coefficient is nearly zero. In fact, Kramer and Lampe [8] find that the BLM scale and the NLO PQCD predictions give a consistent description of the LEP 2-jet and 3-jet data for $0.14>y>0.05$ at the $Z$. Neglecting possible uncertainties due to hadronization effects, this allows a determination of $\alpha_{s}$ with remarkably small error: [8] $\alpha_{\overline{\mathrm{MS}}}\left(M_{z}\right)=0.107 \pm 0.003$, which corresponds to $\Lambda_{\overline{\mathrm{MS}}}^{(5)}=100 \pm 20 \mathrm{MeV}$.

The central principle we have used in our analysis is the fact that vacuum polarization contributions are summed by the running coupling constant in gauge theory. The argument of the running coupling constant is then fixed by the requirement that all fermion vacuum polarization is resummed into $\alpha_{s}$, rather than appear in the coefficients. The fact that NLO correction to the scale $Q^{*}$ is proportional to $\beta_{1}$ is consistent with the Peterman-Stückelberg 
renormalization group analysis and is crucial for applying this method to higher order. We have also seen that this scale-setting procedure leads to correct expansion coefficients in the conformal limit, since the beta-function dependence has been resummed into the renormalization scales. The same procedure can be applied to multi-scale problems; in general, the commensurate scales $Q^{*}, Q^{* *}$, etc. will depend on all of the available scales.

\section{ACKNOWLEDGEMENTS}

We wish to thank G. Bodwin, P. Burrows, L. Dixon, G. Grunberg, P. Huet, G. Ingelman, A. Kataev, G. Kramer, B. Lampe, G. P. Lepage, P. Mackenzie, H. Masuda, D. Müller, M. Peskin, M. Samuel, E. Sather, W. K. Wong, and P. Zerwas for helpful conversations. This work is supported in part by the Department of Energy, contract DE-AC03-76SF00515 and contract DE-FG02-93ER-40762.

\section{APPENDIX}

In this Appendix we present the results of applying the next-to-leading order BLM procedure to relate the five known effective charges $\alpha_{R}(Q), \alpha_{\tau}(Q), \alpha_{g_{1}}(Q) \alpha_{F_{3}}(Q)$ and $\alpha_{F_{1}}(Q)$. Notice that in principle $\alpha_{\tau}(Q)$ is meaningful only for the three-flavor case and for $Q=M_{\tau}$. However, here we will use its analytical expression and leave $Q$ and $f$ dependence explicit. We will leave the scale $Q^{* * *}$ in the following formulas unspecified. This scale in general can be chosen to be $Q^{* *}$, or in the absence of the NLO term, $Q^{*}$. We use $\operatorname{Tr}\left(t^{a} t^{b}\right)=\frac{1}{2} \delta^{a b}$ for the normalization of the trace generators in this Appendix.

$\underline{\alpha_{R}(Q) \text { in terms of } \alpha_{\tau}(Q)}$.

$$
\begin{aligned}
\frac{\alpha_{R}(Q)}{\pi} & =\frac{\alpha_{\tau}\left(Q^{*}\right)}{\pi}+\left(\frac{11}{144}-\frac{1}{6} \zeta_{3}\right) \frac{d^{a b c} d^{a b c}}{C_{F} N} \frac{\left(\sum_{f} Q_{f}\right)^{2}}{\sum_{f} Q_{f}^{2}}\left(\frac{\alpha_{\tau}\left(Q^{* * *}\right)}{\pi}\right)^{3} \\
\ln \left(Q^{*} / Q\right) & =\frac{19}{24}+\frac{169}{1152}\left(\frac{11}{3} C_{A}-\frac{2}{3} f\right) \frac{\alpha_{\tau}(Q)}{\pi}
\end{aligned}
$$


$\underline{\alpha_{R}(Q) \text { in terms of } \alpha_{g_{1}}(Q) .}$

$$
\begin{aligned}
\frac{\alpha_{R}(Q)}{\pi}= & \frac{\alpha_{g_{1}}\left(Q^{*}\right)}{\pi}+\frac{3}{4} C_{F}\left(\frac{\alpha_{g_{1}}\left(Q^{* *}\right)}{\pi}\right)^{2} \\
& +\left[\frac{9}{16} C_{F}^{2}+\left(\frac{11}{144}-\frac{1}{6} \zeta_{3}\right) \frac{d^{a b c} d^{a b c}}{C_{F} N} \frac{\left(\sum_{f} Q_{f}\right)^{2}}{\sum_{f} Q_{f}^{2}}\right]\left(\frac{\alpha_{g_{1}}\left(Q^{* * *}\right)}{\pi}\right)^{3}, \\
\ln \left(Q^{*} / Q\right)= & -\frac{7}{4}+2 \zeta_{3}+\left(-\frac{11}{96}-\frac{7}{3} \zeta_{3}+2 \zeta_{3}^{2}+\frac{\pi^{2}}{24}\right)\left(\frac{11}{3} C_{A}-\frac{2}{3} f\right) \frac{\alpha_{g_{1}}(Q)}{\pi}, \\
\ln \left(Q^{* *} / Q\right)= & -\frac{233}{216}+\frac{64}{9} \zeta_{3}-\frac{20}{3} \zeta_{5}+\left(-\frac{13}{54}+\frac{2}{9} \zeta_{3}\right) \frac{C_{A}}{C_{F}} .
\end{aligned}
$$

$\underline{\alpha_{R}(Q) \text { in terms of } \alpha_{F_{3}}(Q) .}$

$$
\begin{aligned}
\frac{\alpha_{R}(Q)}{\pi}= & \frac{\alpha_{F_{3}}\left(Q^{*}\right)}{\pi}+\frac{3}{4} C_{F}\left(\frac{\alpha_{F_{3}}\left(Q^{* *}\right)}{\pi}\right)^{2} \\
& +\left\{\frac{9}{16} C_{F}^{2}+\left(\frac{11}{144}-\frac{1}{6} \zeta_{3}\right) \frac{d^{a b c} d^{a b c}}{C_{F} N}\left[\frac{\left(\sum_{f} Q_{f}\right)^{2}}{\sum_{f} Q_{f}^{2}}-f\right]\right\}\left(\frac{\alpha_{F_{3}}\left(Q^{* * *}\right)}{\pi}\right)^{3}, \\
\ln \left(Q^{*} / Q\right)= & -\frac{7}{4}+2 \zeta_{3}+\left(-\frac{11}{96}-\frac{7}{3} \zeta_{3}+2 \zeta_{3}^{2}+\frac{\pi^{2}}{24}\right)\left(\frac{11}{3} C_{A}-\frac{2}{3} f\right) \frac{\alpha_{F_{3}}(Q)}{\pi}, \\
\ln \left(Q^{* *} / Q\right)= & -\frac{233}{216}+\frac{64}{9} \zeta_{3}-\frac{20}{3} \zeta_{5}+\left(-\frac{13}{54}+\frac{2}{9} \zeta_{3}\right) \frac{C_{A}}{C_{F}} .
\end{aligned}
$$

$\underline{\alpha_{R}(Q) \text { in terms of } \alpha_{F_{1}}(Q) .}$

$$
\begin{aligned}
\frac{\alpha_{R}(Q)}{\pi}= & \frac{\alpha_{F_{1}}\left(Q^{*}\right)}{\pi}+\frac{5}{4} C_{F}\left(\frac{\alpha_{F_{1}}\left(Q^{* *}\right)}{\pi}\right)^{2}+\left\{\left(-\frac{43}{12}-\frac{85}{6} \zeta_{3}+\frac{115}{6} \zeta_{5}\right) C_{A}^{2}\right. \\
& +\left(10+34 \zeta_{3}-\frac{95}{2} \zeta_{5}\right) C_{A} C_{F}+\left(-\frac{113}{16}-\frac{47}{2} \zeta_{3}+35 \zeta_{5}\right) C_{F}^{2} \\
& \left.+\left(\frac{11}{144}-\frac{1}{6} \zeta_{3}\right) \frac{d^{a b c} d^{a b c}}{C_{F} N} \frac{\left(\sum_{f} Q_{f}\right)^{2}}{\sum_{f} Q_{f}^{2}}\right\}\left(\frac{\alpha_{F_{1}}\left(Q^{* * *}\right)}{\pi}\right)^{3}, \\
\ln \left(Q^{*} / Q\right)= & -\frac{17}{12}+2 \zeta_{3}+\left(-\frac{65}{288}-\frac{7}{3} \zeta_{3}+2 \zeta_{3}^{2}+\frac{\pi^{2}}{24}\right)\left(\frac{11}{3} C_{A}-\frac{2}{3} f\right) \frac{\alpha_{F_{1}}(Q)}{\pi} \\
\ln \left(Q^{* *} / Q\right)= & -\frac{187}{120}+\frac{28}{5} \zeta_{3}-4 \zeta_{5}+\left(-\frac{1}{2}-\frac{28}{15} \zeta_{3}+\frac{8}{3} \zeta_{5}\right) \frac{C_{A}}{C_{F}}
\end{aligned}
$$

$\underline{\alpha_{\tau}(Q) \text { in terms of } \alpha_{R}(Q)}$.

$$
\begin{aligned}
\frac{\alpha_{\tau}(Q)}{\pi} & =\frac{\alpha_{R}\left(Q^{*}\right)}{\pi}-\left(\frac{11}{144}-\frac{1}{6} \zeta_{3}\right) \frac{d^{a b c} d^{a b c}}{C_{F} N} \frac{\left(\sum_{f} Q_{f}\right)^{2}}{\sum_{f} Q_{f}^{2}}\left(\frac{\alpha_{R}\left(Q^{* * *}\right)}{\pi}\right)^{3} \\
\ln \left(Q^{*} / Q\right) & =-\frac{19}{24}-\frac{169}{1152}\left(\frac{11}{3} C_{A}-\frac{2}{3} f\right) \frac{\alpha_{R}(Q)}{\pi}
\end{aligned}
$$


$\underline{\alpha_{\tau}(Q) \text { in terms of } \alpha_{g_{1}}(Q)}$.

$$
\begin{aligned}
\frac{\alpha_{\tau}(Q)}{\pi} & =\frac{\alpha_{g_{1}}\left(Q^{*}\right)}{\pi}+\frac{3}{4} C_{F}\left(\frac{\alpha_{g_{1}}\left(Q^{* *}\right)}{\pi}\right)^{2}+\frac{9}{16} C_{F}^{2}\left(\frac{\alpha_{g_{1}}\left(Q^{* * *}\right)}{\pi}\right)^{3} \\
\ln \left(Q^{*} / Q\right) & =-\frac{61}{24}+2 \zeta_{3}+\left(-\frac{301}{1152}-\frac{7}{3} \zeta_{3}+2 \zeta_{3}^{2}+\frac{\pi^{2}}{24}\right)\left(\frac{11}{3} C_{A}-\frac{2}{3} f\right) \frac{\alpha_{g_{1}}(Q)}{\pi}, \\
\ln \left(Q^{* *} / Q\right) & =-\frac{101}{54}+\frac{64}{9} \zeta_{3}-\frac{20}{3} \zeta_{5}+\left(-\frac{13}{54}+\frac{2}{9} \zeta_{3}\right) \frac{C_{A}}{C_{F}} .
\end{aligned}
$$

$\underline{\alpha_{\tau}(Q) \text { in terms of } \alpha_{F_{3}}(Q)}$.

$$
\begin{aligned}
\frac{\alpha_{\tau}(Q)}{\pi}= & \frac{\alpha_{F_{3}}\left(Q^{*}\right)}{\pi}+\frac{3}{4} C_{F}\left(\frac{\alpha_{F_{3}}\left(Q^{* *}\right)}{\pi}\right)^{2} \\
& +\left\{\frac{9}{16} C_{F}^{2}-\left(\frac{11}{144}-\frac{1}{6} \zeta_{3}\right) \frac{d^{a b c} d^{a b c}}{C_{F} N} f\right\}\left(\frac{\alpha_{F_{3}}\left(Q^{* * *}\right)}{\pi}\right)^{3}, \\
\ln \left(Q^{*} / Q\right)= & -\frac{61}{24}+2 \zeta_{3}+\left(-\frac{301}{1152}-\frac{7}{3} \zeta_{3}+2 \zeta_{3}^{2}+\frac{\pi^{2}}{24}\right)\left(\frac{11}{3} C_{A}-\frac{2}{3} f\right) \frac{\alpha_{\tau}(Q)}{\pi}, \\
\ln \left(Q^{* *} / Q\right)= & -\frac{101}{54}+\frac{64}{9} \zeta_{3}-\frac{20}{3} \zeta_{5}+\left(-\frac{13}{54}+\frac{2}{9} \zeta_{3}\right) \frac{C_{A}}{C_{F}} .
\end{aligned}
$$

$\underline{\alpha_{\tau}(Q) \text { in terms of } \alpha_{F_{1}}(Q)}$.

$$
\begin{aligned}
\frac{\alpha_{\tau}(Q)}{\pi}= & \frac{\alpha_{F_{1}}\left(Q^{*}\right)}{\pi}+\frac{5}{4} C_{F}\left(\frac{\alpha_{F_{1}}\left(Q^{* *}\right)}{\pi}\right)^{2} \\
& +\left\{\left(-\frac{43}{12}-\frac{85}{6} \zeta_{3}+\frac{115}{6} \zeta_{5}\right) C_{A}^{2}+\left(10+34 \zeta_{3}-\frac{95}{2} \zeta_{5}\right) C_{A} C_{F}\right. \\
& \left.+\left(-\frac{113}{16}-\frac{47}{2} \zeta_{3}+35 \zeta_{5}\right) C_{F}^{2}\right\}\left(\frac{\alpha_{F_{1}}\left(Q^{* * *}\right)}{\pi}\right)^{3}, \\
\ln \left(Q^{*} / Q\right)= & -\frac{53}{24}+2 \zeta_{3}+\left(-\frac{143}{384}-\frac{7}{3} \zeta_{3}+2 \zeta_{3}^{2}+\frac{\pi^{2}}{24}\right)\left(\frac{11}{3} C_{A}-\frac{2}{3} f\right) \frac{\alpha_{F_{1}}(Q)}{\pi} \\
\ln \left(Q^{* *} / Q\right)= & -\frac{47}{20}+\frac{28}{5} \zeta_{3}-4 \zeta_{5}+\left(-\frac{1}{2}-\frac{28}{15} \zeta_{3}+\frac{8}{3} \zeta_{5}\right) \frac{C_{A}}{C_{F}}
\end{aligned}
$$

$\underline{\alpha_{g_{1}}(Q) \text { in terms of } \alpha_{R}(Q)}$.

$$
\begin{aligned}
\frac{\alpha_{g_{1}}(Q)}{\pi}= & \frac{\alpha_{R}\left(Q^{*}\right)}{\pi}-\frac{3}{4} C_{F}\left(\frac{\alpha_{R}\left(Q^{* *}\right)}{\pi}\right)^{2} \\
& +\left[\frac{9}{16} C_{F}^{2}-\left(\frac{11}{144}-\frac{1}{6} \zeta_{3}\right) \frac{d^{a b c} d^{a b c}}{C_{F} N} \frac{\left(\sum_{f} Q_{f}\right)^{2}}{\sum_{f} Q_{f}^{2}}\right]\left(\frac{\alpha_{R}\left(Q^{* * *}\right)}{\pi}\right)^{3}, \\
\ln \left(Q^{*} / Q\right)= & \frac{7}{4}-2 \zeta_{3}+\left(\frac{11}{96}+\frac{7}{3} \zeta_{3}-2 \zeta_{3}^{2}-\frac{\pi^{2}}{24}\right)\left(\frac{11}{3} C_{A}-\frac{2}{3} f\right) \frac{\alpha_{R}(Q)}{\pi}, \\
\ln \left(Q^{* *} / Q\right)= & \frac{523}{216}+\frac{28}{9} \zeta_{3}-\frac{20}{3} \zeta_{5}+\left(-\frac{13}{54}+\frac{2}{9} \zeta_{3}\right) \frac{C_{A}}{C_{F}} .
\end{aligned}
$$


$\underline{\alpha_{g_{1}}(Q) \text { in terms of } \alpha_{\tau}(Q)}$.

$$
\begin{aligned}
\frac{\alpha_{g_{1}}(Q)}{\pi} & =\frac{\alpha_{\tau}\left(Q^{*}\right)}{\pi}-\frac{3}{4} C_{F}\left(\frac{\alpha_{\tau}\left(Q^{* *}\right)}{\pi}\right)^{2}+\frac{9}{16} C_{F}^{2}\left(\frac{\alpha_{\tau}\left(Q^{* * *}\right)}{\pi}\right)^{3}, \\
\ln \left(Q^{*} / Q\right) & =\frac{61}{24}-2 \zeta_{3}+\left(\frac{301}{1152}+\frac{7}{3} \zeta_{3}-2 \zeta_{3}^{2}-\frac{\pi^{2}}{24}\right)\left(\frac{11}{3} C_{A}-\frac{2}{3} f\right) \frac{\alpha_{\tau}(Q)}{\pi}, \\
\ln \left(Q^{* *} / Q\right) & =\frac{347}{108}+\frac{28}{9} \zeta_{3}-\frac{20}{3} \zeta_{5}+\left(-\frac{13}{54}+\frac{2}{9} \zeta_{3}\right) \frac{C_{A}}{C_{F}} .
\end{aligned}
$$

$\underline{\alpha_{g_{1}}(Q) \text { in terms of } \alpha_{F_{3}}(Q)}$.

$$
\begin{aligned}
\frac{\alpha_{g_{1}}(Q)}{\pi} & =\frac{\alpha_{F_{3}}\left(Q^{*}\right)}{\pi}-\left(\frac{11}{144}-\frac{1}{6} \zeta_{3}\right) \frac{d^{a b c} d^{a b c}}{C_{F} N} f\left(\frac{\alpha_{F_{3}}\left(Q^{* * *}\right)}{\pi}\right)^{3}, \\
\ln \left(Q^{*} / Q\right) & =0 .
\end{aligned}
$$

$\underline{\alpha_{g_{1}}(Q) \text { in terms of } \alpha_{F_{1}}(Q)}$.

$$
\begin{aligned}
\frac{\alpha_{g_{1}}(Q)}{\pi}= & \frac{\alpha_{F_{1}}\left(Q^{*}\right)}{\pi}+\frac{1}{2} C_{F}\left(\frac{\alpha_{F_{1}}\left(Q^{* *}\right)}{\pi}\right)^{2} \\
& +\left\{\left(-\frac{43}{12}-\frac{85}{6} \zeta_{3}+\frac{115}{6} \zeta_{5}\right) C_{A}^{2}+\left(10+34 \zeta_{3}-\frac{95}{2} \zeta_{5}\right) C_{A} C_{F}\right. \\
& \left.+\left(-\frac{67}{8}-\frac{47}{2} \zeta_{3}+35 \zeta_{5}\right) C_{F}^{2}\right\}\left(\frac{\alpha_{F_{1}}\left(Q^{* *}\right)}{\pi}\right)^{3}, \\
\ln \left(Q^{*} / Q\right)= & \frac{1}{3}-\frac{1}{9}\left(\frac{11}{3} C_{A}-\frac{2}{3} f\right) \frac{\alpha_{F_{1}}(Q)}{\pi} \\
\ln \left(Q^{* *} / Q\right)= & -\frac{37}{36}+\frac{4}{3} \zeta_{3}+\left(-\frac{8}{9}-5 \zeta_{3}+\frac{20}{3} \zeta_{5}\right) \frac{C_{A}}{C_{F}} .
\end{aligned}
$$

$\underline{\alpha_{F_{3}}(Q) \text { in terms of } \alpha_{R}(Q)}$.

$$
\begin{aligned}
\frac{\alpha_{F_{3}}(Q)}{\pi}= & \frac{\alpha_{R}\left(Q^{*}\right)}{\pi}-\frac{3}{4} C_{F}\left(\frac{\alpha_{R}\left(Q^{* *}\right)}{\pi}\right)^{2} \\
& +\left\{\frac{9}{16} C_{F}^{2}-\left(\frac{11}{144}-\frac{1}{6} \zeta_{3}\right) \frac{d^{a b c} d^{a b c}}{C_{F} N}\left[\frac{\left(\sum_{f} Q_{f}\right)^{2}}{\sum_{f} Q_{f}^{2}}-f\right]\right\}\left(\frac{\alpha_{R}\left(Q^{* * *}\right)}{\pi}\right)^{3}, \\
\ln \left(Q^{*} / Q\right)= & \frac{7}{4}-2 \zeta_{3}+\left(\frac{11}{96}+\frac{7}{3} \zeta_{3}-2 \zeta_{3}^{2}-\frac{\pi^{2}}{24}\right)\left(\frac{11}{3} C_{A}-\frac{2}{3} f\right) \frac{\alpha_{R}(Q)}{\pi}, \\
\ln \left(Q^{* *} / Q\right)= & \frac{523}{216}+\frac{28}{9} \zeta_{3}-\frac{20}{3} \zeta_{5}+\left(-\frac{13}{54}+\frac{2}{9} \zeta_{3}\right) \frac{C_{A}}{C_{F}} .
\end{aligned}
$$


$\underline{\alpha_{F_{3}}(Q) \text { in terms of } \alpha_{\tau}(Q) .}$

$$
\begin{aligned}
\frac{\alpha_{F_{3}}(Q)}{\pi}= & \frac{\alpha_{\tau}\left(Q^{*}\right)}{\pi}-\frac{3}{4} C_{F}\left(\frac{\alpha_{\tau}\left(Q^{* *}\right)}{\pi}\right)^{2} \\
& +\left\{\frac{9}{16} C_{F}^{2}+\left(\frac{11}{144}-\frac{1}{6} \zeta_{3}\right) \frac{d^{a b c} d^{a b c}}{C_{F} N} f\right\}\left(\frac{\alpha_{\tau}\left(Q^{* * *}\right)}{\pi}\right)^{3}, \\
\ln \left(Q^{*} / Q\right)= & \frac{61}{24}-2 \zeta_{3}+\left(\frac{301}{1152}+\frac{7}{3} \zeta_{3}-2 \zeta_{3}^{2}-\frac{\pi^{2}}{24}\right)\left(\frac{11}{3} C_{A}-\frac{2}{3} f\right) \frac{\alpha_{\tau}(Q)}{\pi}, \\
\ln \left(Q^{* *} / Q\right)= & \frac{347}{108}+\frac{28}{9} \zeta_{3}-\frac{20}{3} \zeta_{5}+\left(-\frac{13}{54}+\frac{2}{9} \zeta_{3}\right) \frac{C_{A}}{C_{F}} .
\end{aligned}
$$

$\underline{\alpha_{F_{3}}(Q) \text { in terms of } \alpha_{g_{1}}(Q)}$.

$$
\begin{aligned}
\frac{\alpha_{F_{3}}(Q)}{\pi} & =\frac{\alpha_{g_{1}}\left(Q^{*}\right)}{\pi}+\left(\frac{11}{144}-\frac{1}{6} \zeta_{3}\right) \frac{d^{a b c} d^{a b c}}{C_{F} N} f\left(\frac{\alpha_{g_{1}}\left(Q^{* * *}\right)}{\pi}\right)^{3}, \\
\ln \left(Q^{*} / Q\right) & =0 .
\end{aligned}
$$

$\underline{\alpha_{F_{3}}(Q) \text { in terms of } \alpha_{F_{1}}(Q)}$.

$$
\begin{aligned}
\frac{\alpha_{F_{3}}(Q)}{\pi}= & \frac{\alpha_{F_{1}}\left(Q^{*}\right)}{\pi}+\frac{1}{2} C_{F}\left(\frac{\alpha_{F_{1}}\left(Q^{* *}\right)}{\pi}\right)^{2}+\left\{\left(-\frac{43}{12}-\frac{85}{6} \zeta_{3}+\frac{115}{6} \zeta_{5}\right) C_{A}^{2}\right. \\
& +\left(10+34 \zeta_{3}-\frac{95}{2} \zeta_{5}\right) C_{A} C_{F}+\left(-\frac{67}{8}-\frac{47}{2} \zeta_{3}+35 \zeta_{5}\right) C_{F}^{2} \\
& \left.+\left(\frac{11}{144}-\frac{1}{6} \zeta_{3}\right) \frac{d^{a b c} d^{a b c}}{C_{F} N}\right\}\left(\frac{\alpha_{F_{1}}\left(Q^{* * *}\right)}{\pi}\right)^{3} \\
\ln \left(Q^{*} / Q\right)= & \frac{1}{3}-\frac{1}{9}\left(\frac{11}{3} C_{A}-\frac{2}{3} f\right) \frac{\alpha_{F_{1}}(Q)}{\pi}, \\
\ln \left(Q^{* *} / Q\right)= & -\frac{37}{36}+\frac{4}{3} \zeta_{3}+\left(-\frac{8}{9}-5 \zeta_{3}+\frac{20}{3} \zeta_{5}\right) \frac{C_{A}}{C_{F}}
\end{aligned}
$$

$\underline{\alpha_{F_{1}}(Q) \text { in terms of } \alpha_{R}(Q)}$.

$$
\begin{aligned}
\frac{\alpha_{F_{1}}(Q)}{\pi}= & \frac{\alpha_{R}\left(Q^{*}\right)}{\pi}-\frac{5}{4} C_{F}\left(\frac{\alpha_{R}\left(Q^{* *}\right)}{\pi}\right)^{2}+\left\{\left(\frac{43}{12}+\frac{85}{6} \zeta_{3}-\frac{115}{6} \zeta_{5}\right) C_{A}^{2}\right. \\
& +\left(-10-34 \zeta_{3}+\frac{95}{2} \zeta_{5}\right) C_{A} C_{F}+\left(\frac{163}{16}+\frac{47}{2} \zeta_{3}-35 \zeta_{5}\right) C_{F}^{2} \\
& \left.-\left(\frac{11}{144}-\frac{1}{6} \zeta_{3}\right) \frac{d^{a b c} d^{a b c}\left(\sum_{f} Q_{f}\right)^{2}}{C_{F} N}\right\}\left(\frac{\alpha_{R}\left(Q^{* * *}\right)}{\pi}\right)^{3}, \\
\ln \left(Q^{*} / Q\right)= & \frac{17}{12}-2 \zeta_{3}+\left(\frac{65}{288}+\frac{7}{3} \zeta_{3}-2 \zeta_{3}^{2}-\frac{\pi^{2}}{24}\right)\left(\frac{11}{3} C_{A}-\frac{2}{3} f\right) \frac{\alpha_{R}(Q)}{\pi} \\
\ln \left(Q^{* *} / Q\right)= & \frac{51}{40}+\frac{8}{5} \zeta_{3}-4 \zeta_{5}+\left(-\frac{1}{2}-\frac{28}{15} \zeta_{3}+\frac{8}{3} \zeta_{5}\right) \frac{C_{A}}{C_{F}}
\end{aligned}
$$


$\underline{\alpha_{F_{1}}(Q) \text { in terms of } \alpha_{\tau}(Q)}$.

$$
\begin{aligned}
\frac{\alpha_{F_{1}}(Q)}{\pi}= & \frac{\alpha_{\tau}\left(Q^{*}\right)}{\pi}-\frac{5}{4} C_{F}\left(\frac{\alpha_{\tau}\left(Q^{* *}\right)}{\pi}\right)^{2} \\
& +\left\{\left(\frac{43}{12}+\frac{85}{6} \zeta_{3}-\frac{115}{6} \zeta_{5}\right) C_{A}^{2}+\left(-10-34 \zeta_{3}+\frac{95}{2} \zeta_{5}\right) C_{A} C_{F}\right. \\
& \left.+\left(\frac{163}{16}+\frac{47}{2} \zeta_{3}-35 \zeta_{5}\right) C_{F}^{2}\right\}\left(\frac{\alpha_{\tau}\left(Q^{* * *}\right)}{\pi}\right)^{3}, \\
\ln \left(Q^{*} / Q\right)= & \frac{53}{24}-2 \zeta_{3}+\left(\frac{143}{384}+\frac{7}{3} \zeta_{3}-2 \zeta_{3}^{2}-\frac{\pi^{2}}{24}\right)\left(\frac{11}{3} C_{A}-\frac{2}{3} f\right) \frac{\alpha_{\tau}(Q)}{\pi} \\
\ln \left(Q^{* *} / Q\right)= & \frac{31}{15}+\frac{8}{5} \zeta_{3}-4 \zeta_{5}+\left(-\frac{1}{2}-\frac{28}{15} \zeta_{3}+\frac{8}{3} \zeta_{5}\right) \frac{C_{A}}{C_{F}}
\end{aligned}
$$

$\underline{\alpha_{F_{1}}(Q) \text { in terms of } \alpha_{g_{1}}(Q)}$.

$$
\begin{aligned}
\frac{\alpha_{F_{1}}(Q)}{\pi}= & \frac{\alpha_{g_{1}}\left(Q^{*}\right)}{\pi}-\frac{1}{2} C_{F}\left(\frac{\alpha_{g_{1}}\left(Q^{* *}\right)}{\pi}\right)^{2} \\
& +\left\{\left(\frac{43}{12}+\frac{85}{6} \zeta_{3}-\frac{115}{6} \zeta_{5}\right) C_{A}^{2}+\left(-10-34 \zeta_{3}+\frac{95}{2} \zeta_{5}\right) C_{A} C_{F}\right. \\
& \left.+\left(\frac{71}{8}+\frac{47}{2} \zeta_{3}-35 \zeta_{5}\right) C_{F}^{2}\right\}\left(\frac{\alpha_{g_{1}}\left(Q^{* * *}\right)}{\pi}\right)^{3}, \\
\ln \left(Q^{*} / Q\right)= & -\frac{1}{3}+\frac{1}{9}\left(\frac{11}{3} C_{A}-\frac{2}{3} f\right) \frac{\alpha_{g_{1}}(Q)}{\pi}, \\
\ln \left(Q^{* *} / Q\right)= & -\frac{61}{36}+\frac{4}{3} \zeta_{3}+\left(-\frac{8}{9}-5 \zeta_{3}+\frac{20}{3} \zeta_{5}\right) \frac{C_{A}}{C_{F}}
\end{aligned}
$$

$\underline{\alpha_{F_{1}}(Q) \text { in terms of } \alpha_{F_{3}}(Q)}$.

$$
\begin{aligned}
\frac{\alpha_{F_{1}}(Q)}{\pi}= & \frac{\alpha_{F_{3}}\left(Q^{*}\right)}{\pi}-\frac{1}{2} C_{F}\left(\frac{\alpha_{F_{3}}\left(Q^{* *}\right)}{\pi}\right)^{2}+\left\{\left(\frac{43}{12}+\frac{85}{6} \zeta_{3}-\frac{115}{6} \zeta_{5}\right) C_{A}^{2}\right. \\
& +\left(-10-34 \zeta_{3}+\frac{95}{2} \zeta_{5}\right) C_{A} C_{F}+\left(\frac{71}{8}+\frac{47}{2} \zeta_{3}-35 \zeta_{5}\right) C_{F}^{2} \\
& \left.-\left(\frac{11}{144}-\frac{1}{6} \zeta_{3}\right) \frac{d^{a b c} d^{a b c}}{C_{F} N} f\right\}\left(\frac{\alpha_{F_{3}}\left(Q^{* * *}\right)}{\pi}\right)^{3}, \\
\ln \left(Q^{*} / Q\right)= & -\frac{1}{3}+\frac{1}{9}\left(\frac{11}{3} C_{A}-\frac{2}{3} f\right) \frac{\alpha_{F_{3}}(Q)}{\pi}, \\
\ln \left(Q^{* *} / Q\right)= & -\frac{61}{36}+\frac{4}{3} \zeta_{3}+\left(-\frac{8}{9}-5 \zeta_{3}+\frac{20}{3} \zeta_{5}\right) \frac{C_{A}}{C_{F}} .
\end{aligned}
$$




\section{REFERENCES}

* We thank A. Kataev for an illuminating discussion on this point.

$\dagger$ The commensurate ratio $\lambda_{A / V}=Q_{A} / Q_{V}$ differs from the often-called CelmasterGonsalves relation [4, 6, 24,25] for the scales $\Lambda_{A} / \Lambda_{V}$. As discussed in [13, a shift in the renormalization scale as given by $\Lambda_{A} / \Lambda_{V}$ leads to a vanishing NLO coefficient, which is equivalent to the $\mathrm{FAC}$ criterion. In contrast, in the BLM approach, only the $f$ part vanishes.

$\ddagger$ Celmaster and Stevenson [26] have observed that the NLO coefficient $r_{1}^{B / A}$ in an expansion series such as Eq. (2.5) depends on the quantity $A$ used in the right-hand-side, and they have expressed concern that this fact implies a "scheme dependence" of the BLM procedure. This is obviously not a defect: when one expands an observable $B$ in terms of different observables $A$, one clearly obtains different coefficients.

$\S$ We thank Patrick Huet and Eric Sather for conversations on this point.

** The extension of the BLM procedure to higher orders has also been discussed recently by Grunberg and Kataev [17] and by Samuel and Surguladze [2].

I† We must exclude from the analysis the potential $f$ dependence in the NNLO term induced by light-by-light diagrams. These diagrams are finite and do not participate in the renormalization of the running coupling constant. As a convention, the coefficient $D_{12}$ in Eq. (3.1) will include only the $f$ dependence from the running of the coupling constant. The extra $f$-dependent terms from light-by-light scattering diagrams will be considered as part of the $C_{12}$. This separation is straightforward in the practical examples considered in this paper.

$\ddagger \ddagger$ We thank Dieter Müller for discussions on this point.

[1] S. G. Gorishny, A. L. Kataev and S. A. Larin, Phys. Lett. B259, 144 (1991). 
[2] L. R. Surguladze and M. A. Samuel, Phys. Lett. B309, 157 (1993); Phys. Rev. Lett. 66, 560 (1991), 66, 2416(E) (1991).

[3] P. Nason, S. Dawson, and R. K. Ellis, Nucl. Phys. B303, 607 (1988); Nucl. Phys. B327, 49 (1989).

[4] D. T. Barclay, C. J. Maxwell and M. T. Reader, preprint UICHEP-TH-93-14.

[5] G. Grunberg, Phys. Lett. B95, 70 (1980); Phys. Lett. B110, 501 (1982); Phys. Rev. D29, 2315 (1984).

[6] P. M. Stevenson, Phys. Lett. B100, 61 (1981); Phys. Rev. D23, 2916 (1981); Nucl. Phys. B203, 472 (1982); Nucl. Phys. B231, 65 (1984).

[7] S. J. Brodsky, G. P. Lepage and P. B. Mackenzie, Phys. Rev. D28, 228 (1983).

[8] G. Kramer and B. Lampe, Zeit. Phys. A339, 189 (1991).

[9] P. N. Burrows and H. Masuda, preprint SLAC-PUB-6394 (1993).

[10] S. J. Brodsky and H. J. Lu, SLAC-PUB-6000 (1993).

[11] E. C. G. Stückelberg and A. Peterman, Helv. Phys. Acta 26, 499 (1953), A. Peterman, Phys. Rept. 53C, 157 (1979).

[12] A preliminary report of this work appears in S. J. Brodsky and H. J. Lu, Invited talk presented at Leipzig Workshop on Quantum Field Theoretical Aspects of High Energy Physics, Bad Frankenhausen, Germany, September 20-24, 1993. SLAC-PUB-6389.

[13] S. J. Brodsky and H. J. Lu, Phys. Rev. D48, 3310 (1993).

[14] G. P. Lepage, P. B. Mackenzie, Phys. Rev. D48, 2250 (1993).

[15] W. Fischler, Nucl. Phys. B129, 157 (1977), A. Billoire, Phys. Lett. B 92, 343 (1980), W. Buchmuller, G. Grunberg and S.H.H. Tye, Phys. Rev. Lett. 45, 103 (1980); 45, $587(\mathrm{E})(1980)$. 
[16] W. K. Wong, et al., (in preparation).

[17] G. Grunberg and A.L. Kataev Phys. Lett. B279, 352 (1992). G. Grunberg, Phys. Rev. D46, 2228 (1992). J. Chyla and A. L. Kataev, Phys. Lett. B297, 385 (1992). A. Kataev, CERN-TH.6485 (1992), published in the Proceedings of the XXVIIth Recontre de Moriond, ed. J. Tran Than Van (1992).

[18] S. A. Larin, T. van Ritbergen and J.A.M. Vermaseren, Phys. Lett. B320, 159 (1994).

[19] S. A. Larin and J.A.M. Vermaseren, Phys. Lett. B259, 345 (1991).

[20] J. Ellis and M. Karliner, Phys. Lett. B313, 131 (1993).

[21] S. A. Larin, F. V. Tkachov and J.A.M. Vermaseren, Phys. Rev. Lett. 66, 862 (1991).

[22] D. J. Broadhurst and A. L. Kataev, Phys. Lett. B315, 179 (1993).

[23] A. H. Mueller, Phys. Lett. B308, 355 (1993).

[24] W. Celmaster and R.J. Gonsalves, Phys. Rev. D20, 1420 (1979).

[25] A. Dhar and V. Gupta, Phys. Rev. D29, 2822 (1984).

[26] W. Celmaster and P.M. Stevenson, Phys. Lett. B125, 493 (1983). 
This figure "fig1-1.png" is available in "png" format from: http://arxiv.org/ps/hep-ph/9405218v1 